
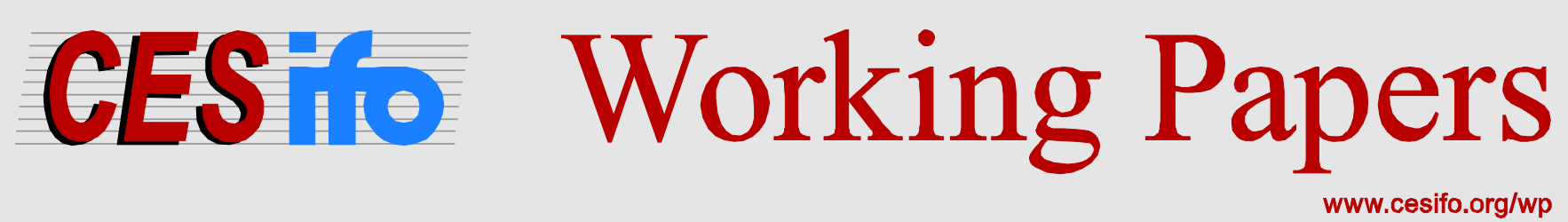

\title{
Inefficient School Choice in a Long-Run Urban Equilibrium
}

\author{
Ulrich Kamecke
}

CESIFO WORKING PAPER NO. 4969

CATEGORY 13: BEHAVIOURAL ECONOMICS

SEPTEMBER 2014

An electronic version of the paper may be downloaded

- from the SSRN website:

- from the RePEc website:

WwW.SSRN.com

- from the CESifo website:

Www.RePEc.org

www.CESifo-group.org/wp

\section{CESifo}




\title{
Inefficient School Choice in a Long-Run Urban Equilibrium
}

\begin{abstract} known (social) differences of the applicants.

JEL-Code: C780, H420, R230. seggregation.

\author{
Ulrich Kamecke \\ Economics / Competition Policy \\ Humboldt-University Berlin \\ Spandauer Str. 1 \\ Germany - 10178 Berlin \\ kamecke@wiwi.hu-berlin.de
}

We model centralized school matching as a second stage of a simple Tiebout-model and show that the two most discussed mechanisms, the deferred acceptance and the Boston algorithm, both produce inefficient outcomes and that the Boston mechanism is more efficient than deferred acceptance. This advantage vanishes if the participants get to know their priorities before they submit their preferences. Moreover, the mechanism creates artificial social segregation at the cost of the disadvantaged if the school priorities are based on ex ante

Keywords: school choice, deffered acceptance, Boston mechanism, Tiebout-hypothesis, social

August 2014 


\section{Introduction}

Matching matters. Not only marriage can make or ruin a man's life, but so can the choice of the right or wrong school, university, job or house. At first sight these matching problems seem very different in nature. While some of them, like most housing or job matching problems, are solved quite satisfactorily in more or less frictionless markets, others seem to inevitably generate chaotic conditions which cannot be eliminated without the help of a central matching authority.

It is widely accepted that the crucial difference between problematic matching problems and those solved satisfactorily in decentralized matching markets is the availability of sufficient flexible means to transfer the utility from one market side to the other. Thus we are faced with two separate branches of research on matching games. On the one hand there is a huge literature on matching in housing or labor markets with side payments, in which perfect information together with a flexible price system would allow an efficient perfectly competitive solution ${ }^{1}$, while search or other frictions may distort the market outcome and hence call for political activities. ${ }^{2}$

On the other hand, there is an unrelated literature on the allocation problems that arise in student or school matching problems, where side payments are not available so that there is no price mechanism to organize the market. Roth (1984) was the first to discuss the role of centralized authorities for the performance of such matching markets. He described the problems caused by the unravelling of the search over time in the market for medical interns and attributed the success of the centralized matching procedure, the NRMP, which was introduced to solve the allocation problem, to the stability of the

\footnotetext{
${ }^{1}$ The linear assignment problem with a nice perfectly competitive solution was presented by Shapley and Shubik (1974). Roth and Sotomayor (1990, part III) present results from several papers which show that many of the properties of this special model generalize to models with non-linear transfers and many-to-one matching problems.

${ }^{2} \mathrm{As}$ it is not the focus of this paper we do not try to give an adequate introduction to the literature on search and matching markets, but only refer to the scientific background paper "The Prize in Economic Sciences 2010 - Advanced Information" compiled by the Economic Sciences Prize Committee of the Royal Swedish Academy of Sciences.
} 
outcomes constructed by this algorithm. Later Roth (1991) confirmed this intuition with the help of a comparison of seven British matching authorities showing that those which did not construct stable solutions but allowed simple manipulation by blocking pairs did not succeed to eliminated the chaotic search frictions. Following these seminal papers several authors identified further matching problems in which the lack of a price system caused various inefficiencies in markets with or without a central authority ${ }^{3}$.

In this context also the school choice problem received considerable attention. The matching of pupils to schools is usually considered as an important political issue, because parents feel very strongly about the education of their children and articulate their disappointment about the outcome of a school matching very loud and clearly. Abdulkadiroglu and Sönmez (2003) present an abstract formulation of the school choice problem and an algorithm to construct the efficient stable solution, while Abdulkadiroglu et.al. (2005a) and (2005b) describe and compare the performance of the two most popular school matching procedures used in the United States.

These papers are typical for the market design approach established for matching problems without side payments ${ }^{4}$. They identify properties of a desirable solution, describe the procedures used to solve a real matching problem, compare the outcome of the real institution with the ideal solution, and propose mechanisms which would produce better outcomes. This way they helped improve the performance of several matching markets including those which assign graduates of law, psychology or medicin to their first jobs, students and children to schools and universities, and even organ transplants to potential receivers.

\footnotetext{
${ }^{3}$ Roth and Xing (1994) present further matching problems which unravel over time. Unravelling problems in the market for law clerks are described in Avery et.al. (2001). In Germany the matching of medical students to universities is run by a complicated centralized matching procedure discussed in Braun et.al. (2010). Roth et.al. (2004,2005) even discuss the efficient assignment of kidney donors to transplant patients.

${ }^{4}$ Note that Roth (2008) does not only summarize the success story of these applications of game theory, but also mentions (p.8-9) the worries about the long run efficiency in the market for interns which we discuss below.
} 
However, concentrated on the last difficult step of these allocation problems the analysis in all these models neglects that money would, in principle, allow to transfer utility also in these applications, and that the lack of a price system is usually justified by informational or legal barriers. In the market for medical interns or judicial clerks, for instance, the available transfer payments are not sufficiently flexible as a competitive price system would have to produce personalized wage offers based on information which is unavailable to the market participants. In other markets like those of the public education system competitive prices are often considered as unfair and therefore as politically unacceptable. Even in marriage the distribution of the gains can be shared between the spouses with the help of explicit or implicit side payments.

If such side payments are feasible but fixed ex ante the matching mechanism is nothing but a complicated rationing scheme which is used after the transfers are already fixed or for which they are determined in a bargaining stage later. Many problems in decentralized matching markets, in particular the unravelling of the outcome over time, can be interpreted as a rush for the rents that remain whenever a market does not reach a competitive equilibrium. These rents do not disappear if the pure matching problem is solved efficiently. They rather go to some of the market participants and may distort their long run incentives considerably.

If we include the distribution of long run rents into the analysis, stability and even ex post efficiency of the matching problem is not as desirable for a satisfactory performance as it appears if one concentrates on the matching outcome alone. Kamecke (1989) showed that the market participation constraint may already be distorted if the transfer payments are determined before the efficient matching is implemented. Even if the subsequent paper by Bulow and Levin (2006) demonstrates that this inefficiency depends on the exact formulation of the matching model and that it almost vanishes if the model allows a little bit more competition, one would expect similar 
problems to appear in matching situations in which there is not even an incomplete price mechanism at work.

In this paper we address the question of the long run efficiency of matching mechanisms in the context of a simple school matching problem. For this purpose we combine a standard rent offer equilibrium in a simple location choice model with a standard school choice solution of a simple matching model. Our schools differ only by their location and the households prefer schools, because their children have to bear higher transportation costs if they live further away from their schools. In the long run the households choose their locations freely and the higher value of the locations close to the schools is reflected by higher equilibrium prices of these locations. We will show that in this environment both, the stable and the Boston matching mechanism produce inefficient allocations and that the Boston allocation is less inefficient than the stable allocation.

With our model we combine two strands of literature. Our long run model is a very simple urban economy in which identical mobile households consume land and a local public service with rivalry in consumption. The capacities of the schools are fixed and there are enough places for all households, but the capacities are distributed such that there is excess supply of schools in the center and excess demand for those in the outskirts. Our short run model, on the other hand, is a simple assignment problem in which the children of the households are matched to a school with a centralized matching mechanism.

Our long run model with land and schooling does not have a natural source of market failure so that it is not surprising that it is possible to decentralize the efficient allocation in a competitive market system. If competitive prices can form for both, school capacities and land, the efficient allocation is reached as a simple bid-rent equilibrium in the sense of Alonso (1964). In order to generate a short run matching problem we therefore impose the natural political restriction that schooling is costless (or that school fees are identical for all children). Under this restriction the value of 
the public service is capitalized in the housing prices ${ }^{5}$. In the light of the Tiebout-hypothesis, it is therefore not surprising that the efficient allocation can still be reached if we allow a complicated location dependent housing price mechanism, or if we introduce a local authority which solves the school matching problem with the ex ante efficient location dependent matching mechanism.

It is the central point of our paper that long run inefficiencies appear as soon as the short run matching mechanism depends on the household preferences, and that these inefficiencies vary with the matching mechanism used. This is an important observation, because the introduction of 'participatory' elements into the school matching mechanism is the central concern of the school choice literature. In the first paragraph of their seminal paper Abdulkadiroğlu and Sönmez (2003) state that "Wealthy parents already have school choice, because they can afford to move to an area with good schools, or they can enroll their child in a private school. Parents without such means, until recently, had no choice of school, and had to send their children to schools assigned to them by the district". Then they concentrate on the discussion of criteria which a participatory matching mechanism should have such as Pareto efficiency, strategy proofness, envy freeness and - if the preferences of the schools are also considered - stability of the match. The result of their analysis is a recommendation how to introduce elements of household preferences into the school matching mechanism without violating fundamental requirements of fairness and efficiency, but this recommendation ignores the long run efficiency of, for instance, the residential housing market.

The reason for the long run market failure in our model is closely related to the discussion of cardinal utility considerations in school matching. In a recent paper Abdulkadiroğlu, Che and Yasuda (2011) demonstrate that there

\footnotetext{
${ }^{5}$ Capitalization of local public services as a mode of the Tiebout (1956) hypothesis models was first proposed by Oates (1969), even though efficiency of these equilibria must be considered an exception as pointed out by Bewley (1981).
} 
is a natural conflict between the strategy proofness of the DA mechanism and the sensitivity to intensities of cardinal preferences which, for instance, the Boston mechanism incorporates better than DA. In the DA algorithm every household submits a preference ordering to the matching authority. The households are tentatively assigned to their first choices and if a school is over-demanded the households with the lowest priorities are rejected and have to turn to their next best alternatives. ${ }^{6}$ The tentative matching generates a Vickrey-property of this algorithm, because unsuccessful applicants can always turn to their next best alternatives later without diminishing their success probabilities for these alternatives by waiting in vain.

This is very different in the Boston mechanism (BM), where such a tentative matching is not used. Instead BM matches as many applicants as possible in each round and rejects only those in over-demanded schools who then have to apply for the remaining capacities. Consequently over-demanded schools may be filled already in the first round, creating an incentive to drop high ranked over-demanded schools from the list in order to improve the chance to get a place in a less over-demanded lower ranked school in an earlier round of the mechanism. This incentive to misrepresent preferences makes rational play in the BM very difficult and has therefore usually been considered as a major drawback of the BM.

Abdulkadiroglu et.al. (2011) readdress this issue. They argue that strategy proofness means that the participants have no incentive to reveal the intensity of their preferences. They propose a model in which all households have the same ranking of schools but different cardinal von NeumannMorgenstern utility values which they aggregate ex ante with the help of a symmetric priority lottery. In this model they show that the households with less intense preferences are the first to omit an over-demanded school from

\footnotetext{
${ }^{6}$ If all schools rank the households in the same order the households with the highest priorities get their first choices, while households with lower priorities have to take what's left - so that they get exactly the same as in the serial dictatorship (if the same priorities are used). Pareto improvements for the households as computed by the top-trading cycles algorithm are not possible in our model.
} 
their list in the BM. This way they generate a framework in which ex ante efficiency distinguishes the matching mechanisms and makes BM perform better than DA even though all matchings are ex post efficient.

By embedding the school matching in a simple spatial model we give more economic meaning to this argument. In the second stage of our model households have chosen their location and prefer closer schools because it is more costly for their children to travel from home to distant schools. These transportation costs serve as a simple cardinal measure for the utility loss. Moreover, the spatial structure of our model creates a natural preference ranking which differs between households. In our first stage a perfectly competitive housing market endogenizes the school rankings of the households. In this stage we assume identical, mobile households thereby generating an outcome which gives the same long run expected utility to everybody, while the short run utility differences at different locations are reflected in the equilibrium prices.

This way we reestablish the major result by Abdulkadiroglu et.al. (2011) in a more general and (in our opinion) more natural economic framework. We demonstrate that short run efficiency allows for many different outcomes in the matching market so that the usual arguments in favor of stable or undominated solutions apply. Long run efficiency, on the other hand, requires the minimization of total transportation costs so that it is inefficient to fill the school capacities with the children from symmetric closest neighborhoods of the schools. Instead applicants from a neighborhood with over-demanded schools should receive preferred access at the neighboring locations and we will show, that this task is not solved by DA nor by BM, and that BM outperforms DA systematically. Finally, we will also show that this advantage of BM disappears if the households know their priorities before they participate in the short run mechanism, because the incentive to reveal the intensity of the preferences depends crucially on the uncertainty involved in this lottery, and that both BM and DA create artificial social segregation if the priorities 
are known already before the housing market clears.

For this purpose we proceed as follows. In section 2 we present a simple limit model with a continuum of households and locations, and with finitely many schools distributed over the city such that there is excess demand for schooling in the outskirts and excess supply towards the center. In section 3 we discuss the efficient outcome in this model and in sections 4 and 5 we characterize the outcomes of our two-stage allocation mechanism if a stable solution is implemented with DA and if BM is used to assign children to schools. In section 6 we discuss the consequences of different assumptions on the information asymmetries in our model. The final section concludes with an outlook on further potential applications of our modelling approach.

\section{The model}

In order to embed our school matching model in a simple general equilibrium framework we use the following spatial economy. A linear city consists of a continuum of uniformly distributed identical houses which are characterized by their location $l \in[0 ; 1]$. Houses are public property. They are offered at the competitive prices $p(l)$, and the housing revenues together with a lump sum tax (or transfer) $\tau$ constitute the public revenues $B=\int_{0}^{1} p(l) d l+\tau$ which are used to finance the schooling expenses.

The houses are bought by a continuum of households $h \in \mathcal{H}$ endowed with a probability measure $\lambda$. Both, the set of households and the set of houses are assumed to have measure one so that a measure preserving ${ }^{7}$ mapping of households to locations is feasible. Each household has a child which has to be educated in one of the schools in town. Education is costly and expenses $c_{h}$ needed to educate a child vary with the child's ability. We assume that the household characteristic $c_{h}$ is uniformly distributed on $[0 ; 1]$, independent of the school, and (for most of the paper) unobserved by the

\footnotetext{
${ }^{7}$ For measure preserving mappings as a natural generalization of a matching for a continuum of agents see Kaneko and Wooders (1986).
} 
households. Further, we assume that all households are ex ante identical and choose their residential locations without knowing their children's $c_{h}$ so that we will have ex ante equal treatment of the households in equilibrium. It is therefore natural to model the location of the household as a random characteristic of the households which is independent of the education cost, so that the households' and their children's characteristics $(l, c)$ are distributed uniformly on $[0 ; 1]^{2}$.

Each child attends one of the $S$ schools $s \in \mathcal{S}$ in town. The quality of the education is the same in every school. School $s$ is characterized by its location $L_{s} \in[0 ; 1]$ with $L_{1}<\ldots<L_{S}$ and by its capacity $K_{s} \in[0 ; 1]$. We assume that the total school capacity just allows to educate all children, $\sum_{s} K_{s}=1$, and that these capacities are concentrated towards the city center which we place at the right boundary of the town, that is at $L=1$. Denote the midpoint between two schools by $\bar{L}_{s}=\left(L_{s}+L_{s+1}\right) / 2$ for $s=1, \ldots, S-1$, let $L_{0}=\bar{L}_{0}=K_{0}=K_{S+1}=0$, and $L_{S+1}=\bar{L}_{S}=1$, and denote the cumulative capacity by $\kappa_{t}=\sum_{s=0}^{t} K_{s}$ for $t=0, \ldots, S$. To define the concentration we consider the rings of households living closest to school $s$ and assume that the schooling capacity per child in these rings of closest distance is increasing as we move from the city limit at $L=0$ towards the city center at $L=1$ :

$$
\frac{K_{s}}{\bar{L}_{s}-\bar{L}_{s-1}}<\frac{K_{s+1}}{\bar{L}_{s+1}-\bar{L}_{s}}
$$

This assumption (1) generates excess demand for schooling at $L=0$ and excess supply at $L=1$ so that some children have to travel towards the city center to fill the excess capacity. Moreover, (1) implies that the cumulated average excess demand at the ring boundaries $\left(\bar{L}_{s}-\kappa_{s}\right)$ is positive and nshaped: it increases up to the location $\bar{s}$ at which $K_{\bar{s}} /\left(\bar{L}_{\bar{s}}-\bar{L}_{\bar{s}-1}\right)<1 \leq$ $K_{\bar{s}+1} /\left(\bar{L}_{\bar{s}+1}-\bar{L}_{\bar{s}}\right)$ and decreases thereafter.

A (complete) matching of households to schools is represented by a 
measurable mapping $\mu: \mathcal{H} \rightarrow \mathcal{S}$. Throughout the paper we concentrate on school choice problems which guarantee a place to every child, that is we assume $\lambda\left(\mu^{-1}(s)\right)=K_{s}$ for every school. Consequently we do not have to specify matchings that allow outside options at this place. In addition, we assume that there is a density function $m(l, c, s)$ which characterizes the mass of households with characteristic $(l, c)$ assigned to school $s$ by

$$
\lambda\left(\left\{h \in \mathcal{H} \mid l_{h} \in L, c_{h} \in C \text { and } \mu(h)=s\right\}\right)=\int_{L} \int_{C} m(l, c, s) d c d l
$$

for all measurable $L \subset[0 ; 1]$ and $C \subset[0 ; 1]$ so that $\int_{0}^{1} \int_{0}^{1} m(l, c, s) d c d l=K_{s}$ holds for all schools $s$. A household $h$ living at $l_{h}$ is assigned to school $s$ with probability $M\left(l_{h}, s\right)=\int_{0}^{1} m\left(l_{h}, c, s\right) d c$.

For a matching $\mu$ school $s$ has to bear the total education $\operatorname{cost} C_{s}=$ $\int_{0}^{1} \int_{0}^{1} c \cdot m(l, c, s) d c d l$. In order to cover these costs the government determines a budget for each school $B_{s}$ in the first stage of the game, which may or may not cover the entire education costs. Throughout the paper we assume that schools care about their profits $C_{s}-B_{s}$ so that they prefer children with low $c_{h}$, and that these preferences determine the households' priorities in the matching mechanism. However, in reality most public schools are not allowed to select their pupils according to their preferences. In order to make our model more realistic we can interpret $c_{h}$ as a random priority ${ }^{8}$ which each household receives in a lottery after he submits his preferences to the matching authority. In this case it does not make sense to assume that the education cost depends on $c_{h}$, but since these costs do not influence the efficiency of the outcome our results are not affected if we replace the school budget constraints by $C_{s}=B_{s}=0$.

\footnotetext{
${ }^{8}$ See, for instance, the mechanisms discussed in Abdulkadiroglu et.al. (2005). The close relationship between models with and without school preferences is also discussed in Abdulkadiroglu and Sönmez (2003, p.31). Our assumption of a uniform ranking of pupils by all schools according to $c_{h}$ simplifies the matching problem considerably. In particular the stable solution constructed by DA and the solution constructed by serial $c_{h}$-dictatorship conincide and there is no room for further improvement if the school preferences don't matter as in Abdulkadiroglu and Sönmez (2003).
} 
Households care about different schools because the transportation to school is costly. In addition to the housing price $p\left(l_{h}\right)$ household $h$ has to pay the linear transportation cost $t \cdot\left|L_{\mu(h)}-l_{h}\right|$ for the distance from his home $l_{h}$ to the location $L_{\mu(h)}$ of the school at which the child living in this household is accepted. Moreover, we assume that every household has to pay the lump sum tax $\tau$ which we introduce to balance budgets so that the total utility loss of $h$ is given by

$$
u_{h}=\tau+p\left(l_{h}\right)+\mu(h) \cdot t \cdot\left|l_{h}-L_{s}\right| .
$$

If the households choose their locations before observing $c_{h}$ they minimize their ex ante expected utility loss

$$
U_{h}=\int_{0}^{1} u_{h} d c=\tau+p\left(l_{h}\right)+\sum_{s=1}^{S} t \cdot\left|l_{h}-L_{s}\right| \cdot M\left(l_{h}, s\right)
$$

The final allocation in our model is constructed in a two stage procedure. In the first stage the housing market clears at equilibrium prices $p^{*}(l)$ and the government determines the budgets $B_{s}$ granted to the schools. In this stage the government determines the $B_{s}$ such that they just cover the ex post equilibrium education costs in all schools, while the households maximize their expected utilities. In the second stage each household submits a complete ranking $R_{h}$ from the set $\Re$ of complete ordered preference lists $R_{h}=\left(R_{h}(1), \ldots R_{h}(S)\right)$ of all $S$ schools $^{9}$, a centralized matching mechanism determines the matching $m(\cdot)$ of children to schools, and (in order to guarantee ex post feasibility of the outcome) the lump sum tax (or subsidy) $\tau$ for the households always balances the total budget, $\tau=\sum_{s}\left(C_{s}-B_{s}\right)-\int_{0}^{1} p(l) d l$. Throughout the paper the (strategy) profile $R: \mathcal{H} \rightarrow \Re$ is assumed to be measurable.

\footnotetext{
${ }^{9}$ As the households do not have outside options it is reasonable to restrict the attention to complete rankings of all $S$ schools. Under our assumption of sufficient capacity this guarantees that every ranking will generate a feasible solution under the matching algorithms discussed in the following.
} 


\section{$3 \quad$ Efficiency}

Free mobility of the households implies that that the ex ante expected equilibrium payments $U_{h}=E u_{h}$ coincide in all locations. We therefore restrict also our efficiency discussion to the attention to outcomes with ex ante identical utilities. Since $U_{h}$ increases with the transportation costs, while the total education costs are not influenced by the matching of children to schools, society should try to select an aggregate transportation cost minimizing matching. In our linear model this minimum is reached whenever no children travel unnecessarily to a school in the "wrong" direction away from the city center. The "capacity ring" matching

$$
m^{*}(l, c, s)= \begin{cases}1 & \text { if } l \in\left[\kappa_{s-1} ; \kappa_{s}\right] \\ 0 & \text { otherwise }\end{cases}
$$

obviously solves this problem by filling the school capacities with households living in capacity rings. The resulting minimal transportation costs are:

$$
T^{*}=t \cdot \sum_{s=1}^{S} \int_{\kappa_{s-1}}^{\kappa_{s}}\left|l-L_{s}\right| d l .
$$

If all school locations $s<S$ satisfy $\kappa_{s} \geq L_{s}$ then every school has to accept some children who travel away from the center and $m^{*}$ is the unique efficient matching. However, if $\kappa_{s}<L_{s}$ some children living on the left side of the school, $l_{h}<L_{s}$, have to move further towards the city center. In this case efficiency requires that $K_{s}$ of the households in the ring $\left[\kappa_{s-1} ; L_{s}\right]$ are assigned to school $s$, and that the rest travels further towards the center. A matching which does not satisfy this condition is inefficient, but it does not matter for efficiency which children have to travel.

Since our simple urban model does not exhibit a natural market failure it is not surprising that the efficient allocations can be decentralized as competitive equilibria with location dependent prices $p^{*}(l)$ for the houses and with school fees $F_{s}^{*}$. Ex ante indifference of the mobile households requires 
that all total payments coincide so that the efficient matching can only be decentralized if

$$
p^{*}(l)+t\left|L_{s}-l\right|+F_{s}^{*}=p_{0}
$$

holds for $\kappa_{s-1} \leq l \leq \kappa_{s}$ and all $s \in\{1, \ldots, S\}$. Optimality of the school choices in (2) implies that $p^{*}(l)$ is continuous at the ring borders $\kappa_{s} \leq \bar{L}_{s}$ (by assumption (1)), so that

$$
F_{s+1}^{*}-F_{s}^{*}=2 \cdot t \cdot\left(\bar{L}_{s}-\max \left\{\kappa_{s}, L_{s}\right\}\right)
$$

must hold for all $s \in\{1, \ldots, S-1\}$. The level of the equilibrium fees $F_{S}^{*}$ and the housing prices $p_{0}$ are undetermined, because higher fees and prices lead to lower $\tau$ and hence to the same allocation. The efficient equilibria are therefore characterized by (3) and (4), if we normalize, for instance, by $F_{S}^{*}=\tau=0$.

Throughout this paper we will assume that schooling is free of charge. In this case the value difference between different school neighborhoods capitalizes in the housing prices so that we could still reach the efficient solution if we can impose school dependent housing prices

$$
\widehat{p}_{s}(l)=p^{*}(l)-F_{s}^{*}
$$

For these housing prices all households are indifferent so that the matching $m^{*}$ is an equilibrium outcome. For this competitive mechanism to work, however, there have to be $S$ different prices at each location.

In order to reach this capitalization equilibrium in a more natural setting with only one discontinuous price function

$$
p^{* *}(l)=p^{*}(l)-F_{s}^{*} \text { for } \kappa_{s-1} \leq l \leq \kappa_{s} \text { for all } s \in\{1, \ldots, S\}
$$

we have to introduce a matching agency which forces households to accept the efficient equal treatment matching $m^{*}$ in the last stage. This matching agency therefore uses a purely location dependent algorithm to assign children to 
schools. In this case the households know to which school the children are sent when they move to their houses so that it is indeed the combination of free (or equal fee) schooling and a preference depending school choice procedure which introduces a second best problem into our model.

Example To demonstrate the problem consider the following example. Suppose there are three schools $s=1,2,3$ located at $L_{s} \in\{1 / 3,2 / 3,1\}$, while the population of pupils is distributed uniformly on the unit interval. All schools are identical in quality and they all have a capacity $K_{s}=1 / 3$. We further assume that the linear transportation costs are given by $t=1$ for each distance unit from the location to the school so that the school located at the center $L_{3}=1$ is under-demanded, while the left school at $L_{1}=1 / 3$ is over-demanded.

The efficient matching in this example is ${ }^{10}$

$$
m^{*}(l, c, s)= \begin{cases}1 & \text { if } l \in[(s-1) / 3 ; s / 3] \\ 0 & \text { otherwise }\end{cases}
$$

with total transportation costs

$$
T^{*}=\int_{0}^{1 / 3}|l| d l+\int_{1 / 3}^{2 / 3}|l-1 / 2| d l+\int_{2 / 3}^{1}|l-3 / 4| d l=1 / 6 .
$$

They measure the efficiency of the allocation since all utilities coincide and since the total education costs are $C=1$ independently of the matching.

As there are no children traveling outward the prices are increasing everywhere so that the efficient allocation is realized with the price function $p^{*}(l)=1 / 6+l$, the school fees $F^{*}=(2 / 3,1 / 3,0)$, and the school budgets $B_{s}=1 / 3$. For these prices and fees every household pays a total of $p_{0}=7 / 6$ for housing and school fees so that the total revenue just covers the education costs of 1 and the total transportation costs of $1 / 6(\tau=0)$.

\footnotetext{
${ }^{10}$ Since no students have to commute from the outer rings to the center this matching is unique.
} 


\section{Deferred Acceptance}

In this section the households' utilities are not observed by the schools or the social planner. Instead the (strict) preference list $R_{h}=\left(R_{h}(1), \ldots, R_{h}(S)\right)$ over schools becomes a strategic choice of each household. In finite models stability of a matching is a well-known concept. A proposed matching can be blocked by a school-household pair if the school prefers the household's child to some of its proposed pupils, while the household prefers the school to the proposed school. A matching is stable if it cannot be blocked, and it is well-known that such a stable outcome can be constructed with the following deferred acceptance (DA) algorithm ${ }^{11}$ :

1. in the first iteration $i=1$ every household applies for one of the places in the first school $R_{h}(1)$ on his preference list; for each school $s$ the applicants are collected in the set $\mathfrak{A}_{s 1}^{S} \cdot{ }^{12}$

2. in iteration $i=1, \ldots$ the critical cost level

$$
c_{s i}^{S}=\max \left\{c \in[0 ; 1] \mid \lambda\left(h \in \mathfrak{A}_{s i}^{S} \mid c_{h} \leq c\right) \leq K_{s}\right\}
$$

is determinedeach for each school; each school $s$ fills the open capacity $K_{s}$ tentatively with the best households from the actual set of applicants $\mathfrak{A}_{s i}^{S}$ and the remaining candidates $\left\{h \in \mathfrak{A}_{s i}^{S} \mid c_{h}>c_{s i}^{S}\right\}$ are rejected;

3. if there are households $h \in \mathfrak{A}_{s i}^{S}$ who were rejected in the present iteration $i$ by the school $s$ which ranked $j_{h i}$-th on their preference list, these households are forwarded as additional applicants to the next school $R_{h}\left(j_{h i}+1\right)$ on their preference lists so that

$$
\mathfrak{A}_{s i+1}^{S}=\left\{h \in \mathfrak{A}^{S} \mid c_{h} \leq c_{s i}^{S}\right\} \cup \bigcup_{t}\left\{h \in \mathfrak{A}^{S} \mid c_{h}>c_{t i}^{S} \text { and } R_{h}\left(j_{h i}+1\right)=s\right\}
$$

\footnotetext{
${ }^{11}$ See Roth and Sotomayor (1991) for a survey of the origins of the algorithm.

${ }^{12}$ In the following the superscript $S$ denotes stability while the subscript $s$ is the variable for the schools.
} 
and the algorithm returns to step 2 setting $i=i+1$; otherwise the algorithm stops with $c_{s \infty}^{S}=c_{s i}^{S}$ and $\mathfrak{A}_{s \infty}^{S}=\mathfrak{A}_{s i}^{S}$.

In the infinite model discussed in this paper stability can be defined in the same way, and our version of DA works also for a continuum of households, even though it does not necessarily converge in finitely many steps. However, measurability of the strategies guarantees that the decreasing sequence of critical costs converge to $c_{s \infty}^{S}=\lim _{i \rightarrow \infty} c_{s i}^{S}$ so that the algorithm converges to a well-defined (tentative) stable limit ${ }^{13}$ which assigns the applicants $h \in$ $\mathfrak{A}_{s \infty}^{S}=\lim _{i \rightarrow \infty} \mathfrak{A}_{s i}^{S}$ to school $s$.

The structural assumption (1) makes it easy to predict the outcome of the deferred acceptance algorithm. For all households it is a dominant strategy to apply at the closest schools first and all schools use the same criterion $c$ to rank their applicants, while (1) guarantees that for large $H$ the schools are less over-demanded as we move towards the city center. We can therefore conclude that the first school $s=1$ will eventually have the strictest entry requirement $c_{1 \infty}^{S}$ and that schools further downtown accept also more and more costly applicants. Rejected applicants have to travel further downtown, and since the critical costs are decreasing a dominant strategy involves listing the closest school towards the city center next (perhaps also after listing some schools further away from the city center which can never be successful after a rejection). The new applicants allow the schools to improve the average cost parameter of their pupils, and again the children with the highest costs are rejected. This way we construct a class of dominant strategies which construct a unique ex post stable matching so that also the schools have no incentive to misrepresent their preferences.

Theorem 1 (Stable solution) The critical cost level for school $s$ is given by $c_{s \infty}^{S}=K_{s} /\left(\bar{L}_{s-1}-\kappa_{s-1}\right)$. The stable matching $\mu^{S}$ assigns the low cost chil-

\footnotetext{
${ }^{13}$ Note that each household can be rejected at most $S$ times so that the total mass of rejections during the procedure is bounded by $S$. If a positive mass $\varepsilon>0$ remained unmatched in the limit, the total mass of rejections would diverge.
} 
dren to their most preferred schools and higher cost children further downtown, so that the corresponding matching density satisfies

$$
m^{S}(l, c, s)= \begin{cases}1 \quad \text { if }\left\{l \in\left[\bar{L}_{s-1} ; \bar{L}_{s}\right) \text { and } c \in\left[0 ; c_{s \infty}^{S}\right]\right\} \\ \text { or }\left\{l \in\left[0 ; \bar{L}_{s}\right] \text { and } c \in\left(c_{s \infty}^{S} ; c_{s+1 \infty}^{S}\right]\right\} \\ 0 \quad \text { otherwise. }\end{cases}
$$

The unique limit allocation constructed by the corresponding two-stage market mechanism is inefficient.

Proof: In the first iteration the households located in the rings $\left(\bar{L}_{s-1} ; \bar{L}_{s}\right)$ select school $s$ as their first choices, and the critical cost levels are set to $c_{s 1}^{S}=\min \left\{K_{s} /\left(\bar{L}_{s}-\bar{L}_{s-1}\right), 1\right\}$ so that monotonicity of the critical costs $c_{11}^{S} \leq \ldots \leq c_{S 1}^{S}$ holds by construction (with a strict inequality as long as $\left.c_{s 1}^{S}<1\right)$.

Suppose that the monotonicity of the critical costs holds in iteration $i$, i.e. $c_{1 i}^{S} \leq \ldots \leq c_{S i}^{S}$ (with a strict inequality as long as $c_{s i}^{S}<1$ ), then it also holds in iteration $i+1$ : if new applicants lead to a decrease of the critical cost level $c_{s i+1}^{S}<c_{s i}^{S}$ then monotonicity implies that these new applicants must have been rejected at a school $s^{\prime}<s$ and hence in particular in school $s-1$ before. Since the critical costs never increase in an iteration, $c_{s i+1}^{S} \leq c_{s i}^{S}$, this implies $c_{s i+1}^{S} \geq c_{s-1 i}^{S} \geq c_{s-1 i+1}^{S}$ so that monotonicity holds also for iteration $i+1$.

Given this monotonicity we can construct the final outcome with the following modified algorithm: after the first iteration, no household will ever turn successfully to $s=1$, so that $c_{1 \infty}^{S}=c_{11}^{S}=K_{1} / \bar{L}_{1}$. The rejected households $h \in\left[0 ; \bar{L}_{1}\right]$ will turn to $s=2$ and decrease the critical cost to $c_{2 \infty}^{S}=K_{2} /\left(\bar{L}_{2}-K_{1}\right)$. All households $h \in\left[0 ; \bar{L}_{2}\right]$ who were rejected by $s \in\{1,2\}$ will eventually turn to $s=3$ and decrease the critical cost to $c_{3 \infty}^{3}=K_{3} /\left(\bar{L}_{3}-K_{1}-K_{2}\right)=K_{3} /\left(\bar{L}_{3}-\kappa_{2}\right)$, and so on. This way we construct the critical costs $c_{s \infty}^{S}$ and the matching $m^{S}$ as defined in the theorem. 
Finally, note that (1) implies that $c_{1 \infty}^{S}<1$ so that there is some $\varepsilon>0$ such that the mass $\varepsilon$ of children travel from their locations $l \in\left(0 ; \varepsilon /\left(1-c_{1 \infty}^{S}\right)\right)$ to some school $s>1$, while another mass $\varepsilon$ of children travel from their locations $l \in\left(\bar{L}_{1}-\varepsilon / c_{1 \infty}^{S} ; \bar{L}_{1}\right)$ to school 1 . Moreover, we can select $\varepsilon$ small enough to garantee that $\bar{L}_{1}-\varepsilon / c_{1 \infty}^{S}>L_{1}$ so that the live to the right of $L_{1}$. If these children exchange their schools the aggregate transportation costs are reduced by more than $\left(\bar{L}_{1}-L_{1}-\varepsilon /\left(2 c_{1 \infty}^{S}\right)\right) \cdot \varepsilon>0$, because each such exchange of children saves the transportation costs spent on traveling away from the city center.

Example In the example introduced above all households with locations $l \in[0 ; 1 / 2)$ prefer the left school, so that the available capacity in the inner ring is filled with the applicants whose costs are below $c_{3 \infty}^{S}=2 / 3$. The households with locations $l \in(1 / 2 ; 5 / 6]$ prefer the middle school so that the critical cost level $c_{2 \infty}^{S}=(2 / 3)(6 / 5)=4 / 5$ fills the school with a mass of $4 / 15$ living in the middle ring and $(4 / 5-2 / 3) / 2=1 / 15$ in the inner ring. The remaining households commute to the center at $l=1$. The stable matching density is therefore given by

$$
m^{S}(l, c, s)= \begin{cases}1 & \text { if } s=1, l \in[0 ; 1 / 2] \text { and } c_{h} \in[0 ; 2 / 3] \\ 1 & \text { if } s=2, l \in[0 ; 1 / 2] \text { and } c_{h} \in[2 / 3 ; 4 / 5] \\ 1 & \text { if } s=2, l \in[1 / 2 ; 5 / 6] \text { and } c_{h} \in[0 ; 4 / 5] \\ 1 & \text { if } s=3, l \in[0 ; 5 / 6] \text { and } c_{h} \in[4 / 5 ; 1] \\ 1 & \text { if } s=3, l \in[5 / 6 ; 1] \\ 0 & \text { otherwise. }\end{cases}
$$

The total transportation costs generated by this stable matching are

$$
\begin{aligned}
T^{S}= & \frac{2}{3} \int_{0}^{1 / 2}|1 / 3-l| d l+\frac{2}{15} \int_{0}^{1 / 2}|2 / 3-l| d l+\frac{4}{5} \int_{1 / 2}^{5 / 6}|2 / 3-l| d l \\
& +\frac{1}{5} \int_{0}^{5 / 6}|1-l| d l+\int_{5 / 6}^{1}|1-l| d l=\frac{28}{135} .
\end{aligned}
$$


They exceed the minimum transportation costs, because $2 / 3$ of the households living in $[1 / 3 ; 1 / 2]$ and $4 / 5$ of those in $[2 / 3 ; 5 / 6]$ should also commute inward to the center instead of outward.

The possibility that the children may have to travel long distance to the school diminishes the value of the left locations. To level this disadvantage the equilibrium prices (calculated again such that $\tau=0$ and $B_{s}=1 / 3$ ) have to reflect the expected travel costs in each location,

$$
p^{S}(l)=1+\frac{28}{135}-E\left[T^{S}(l)\right]
$$

which in turn are determined by

$$
E\left[T^{S}(l)\right]= \begin{cases}23 / 45-l & \text { if } l \in[0 ; 1 / 3] \\ 1 / 15+l / 3 & \text { if } l \in[1 / 3 ; 1 / 2] \\ 11 / 15-l & \text { if } l \in[1 / 2 ; 2 / 3] \\ -5 / 15+3 l / 5 & \text { if } l \in[2 / 3 ; 5 / 6] \\ 1-l & \text { if } l \in[5 / 6 ; 1]\end{cases}
$$

The efficiency loss caused by the stable matching is reflected by the higher price at the school location $s_{1}=1$, where the households don't have to pay any transportation costs. At the other two school locations they pay less than in the efficient matching if they do not have to travel, but there is always a positive fraction of households who have to commute.

\section{The Boston solution}

The Boston mechanism (BM) does not know a tentative matching. It immediately assigns best applicants to best schools so that households who are not successful with their earlier priorities are assigned to schools which are not filled in earlier iterations. Again the individual rankings $R_{h}$ are selected from the set $\Re$ of all $\sum_{k=1}^{S} k$ ! possible preference lists and $R$ is a (measurable) strategy profile. In the following we denote the expected transportation costs 
of household $h$ for a strategy profile $R$ by $T_{h}(R)$, a strategy profile in which household $h$ plays $R_{h}^{\prime}$ instead of $R_{h}$ by $\left(R \mid R_{h}^{\prime}\right)$.

The Boston outcome is constructed with a repetition of the following steps $^{14}$ :

1. in the first iteration $i=1$ every household applies for one of the places in the first school $R_{h}(1)$ on his preference list; for each school $s$ the applicants are collected in the set $\mathfrak{A}_{s 1}^{B}$. The remaining capacity is set to $K_{s 1}^{B}=K_{s}$ and the matching is set to $\mu^{B}(h)=\emptyset$.

2. in iteration $i=1, \ldots, I$ each school $s$ fills the remaining capacity $K_{s i}^{B}$ with the best households from the actual set of applicants $\mathfrak{A}_{s i}^{B}$ : for each school the critical cost level is determined as

$$
c_{s i}^{B}= \begin{cases}0 & \text { if } K_{s i}^{B}=0 \\
\inf \left\{c \mid \lambda\left(\left\{h \in \mathfrak{A}_{s i}^{B} \mid c_{h} \leq c\right\}\right) \geq K_{s i}^{B}\right\} & \left\{\begin{array}{l}
\text { if } \mathfrak{A}_{s i}^{B} \neq \emptyset \\
\text { and } K_{s i}^{B}>0
\end{array}\right. \\
1 & \text { otherwise }\end{cases}
$$

the low cost households are matched to school $s, \mu^{B}(h)=s$ if $h \in$ $\mathfrak{A}_{s i}^{B}$ and $c_{h} \in\left[0 ; c_{s i}^{B}\right]$; the remaining candidates $\left\{h \in \mathfrak{A}_{s i}^{B} \mid c_{h}>c_{s i}^{B}\right\}$ are rejected $^{15}$;

3. if there are rejected households, they are forwarded as applicants to the next school $R_{h}(i+1)$ on their lists so that

$$
\mathfrak{A}_{s i+1}^{B}=\bigcup_{t}\left\{h \in \mathfrak{A}_{t i}^{B} \mid c_{h}>c_{t i}^{B} \text { and } R_{h}(i+1)=s\right\}
$$

and the algorithm returns to step 2 with $K_{s i+1}^{B}=\max \left\{K_{s i}^{B}-\lambda\left(\mathfrak{A}_{s i}^{B}\right), 0\right\}$; otherwise the algorithm stops with a feasible solution.

\footnotetext{
${ }^{14}$ The algorithm is the continuous generalization of the Boston matching scheme as presented, for instance, in Abdulkadiroglu et.al (2005, p.369).

${ }^{15}$ With this formulation we guarantee that finitely many applicants high costs are rejected. This is necessary to destroy an incentive to deviate below.
} 
In every iteration of this algorithm every household is either matched or rejected so that the solution is constructed after at most $S$ iterations. However, this computational simplification comes with a strategic complication as the households now face a tradeoff if they rank over-demanded schools first, because they risk to lose an attractive alternative during the ongoing round. To see this consider the strategies of the households living close to and left of the middle of the town, $l=0.5-\varepsilon$, in our example above. If all households submit their true preferences in BM then the first school is over-demanded while the second school can just accommodate all applicants. So the households living close to $l=0.5-\varepsilon$ are accepted at school 1 with probability $2 / 3$ and have to travel to the center otherwise which generates an expected transportation cost of almost $T=4 / 9$. If they turn immediately to school 2 instead, they are accepted (almost) certainly and therefore expect transportation costs of a little more than $1 / 3$. We can therefore expect that these households are the first to turn to the schools toward the center of the city. This improves the performance of the matching mechanism, because the incentive to turn to a less over-demanded schools is stronger for those households who care less for the over-demanded school. This will lead to a more efficient outcome than the stable matching.

Unfortunately, the efficiency gain of the Boston mechanism is not for free, because the finite Boston game is strategically much more complex than the dominance solvable stable mechanism. In particular, it is easy to see that the game is not dominance solvable. Moreover, the finite Boston game may suffer from a serious coordination problem which may lead to multiple inefficient equilibrium or to non-existence of a pure strategy equilibrium. In our simple limit model most of these problems disappear and all equilibria of the game will turn out to be well-structured, because all remaining strategic difficulties appear in the "Boston scramble", that is the strategic choice of the schools ranked second, third and so on, and this part of the game is irrelevant for the overall welfare as the total transportation costs are not affected by the 
matching realized by the households who are rejected in the first round.

The following theorem shows that the Boston equilibrium exists, that it has the natural ring structure (as long as it is also imposed by efficiency) with increasing success probabilities towards the city center, and that the Boston allocation is in general less inefficient than the stable solution.

Theorem 2 Suppose the households do not know their cost characteristic $c_{h}$ before the application procedure starts, then there is a Boston equilibrium $R^{B}$. Moreover, every Boston equilibrium $R^{B}$ has the following three properties:

- (Decreasing first round excess demand) There is a school $s^{B}>1$ such that $c_{11}^{B}<c_{21}^{B}<\ldots<c_{s^{B} 1}^{B}$ and $c_{s 1}^{B}=1$ for all $s=s^{B} \ldots S$.

- (Ring structure) Define ring borders $b_{s}^{B}$ recursively by $b_{0}^{B}=0$ and $b_{s}^{B}=b_{s-1}^{B}+K_{s} / c_{s 1}^{B}$. Households $h$ living at $l_{h} \in\left[L_{s} ; b_{s}^{B}\right]$ list school $s$ first, that is $R_{h}^{B}(1)=s$, while households $h$ living at $l_{h} \in\left[b_{s-1}^{B} ; L_{s}\right]$ do not lose deviating to the equilibrium strategy $R_{s}^{B}$ of a household who lists school $s$ first, that is, $T_{h}\left(R^{B}\right)=T_{h}\left(R^{B} \mid R_{h}=R_{s}^{B}\right)$ so that in particular $b_{s-1}^{B}>L_{s-1} \Rightarrow R_{h}^{B}(1)=s$ for all households $h$ living at $l_{h} \in\left[b_{s-1}^{B} ; b_{s}^{B}\right]$.

- (Efficiency) A Boston equilibrium allocation is at least as efficient as the stable equilibrium allocations.

The proof of this theorem is rather complicated and therefore deferred to the appendix.

Example In the example introduced above the first two schools are filled in the first round because their capacities are exhausted by the first choice households with locations $l \in[0 ; 1 / 2]$ and $l \in[1 / 2 ; 5 / 6]$ so that unsuccessful households have to travel to school 3 at the center. For households 
$l \in[1 / 2 ; 5 / 6]$ it is obviously better to try to get their first choice school so that $\beta_{2}=5 / 6$. Using $c_{11}^{B}=1 / 3 \beta_{1}$ and $c_{12}^{B}=1 / 3\left(5 / 6-\beta_{1}\right)$ the indifference condition for households at the boundary of the first ring

$$
\left(\beta_{1}-1 / 3\right) \cdot c_{11}^{B}+\left(1-\beta_{1}\right)\left(1-c_{11}^{B}\right)=\left(2 / 3-\beta_{1}\right) \cdot c_{12}^{B}+\left(1-\beta_{1}\right) \cdot\left(1-c_{12}^{B}\right)
$$

yields the ring border and the critical cost levels

$$
\begin{aligned}
\beta_{1} & =\frac{5-\sqrt{5}}{6} \approx 0.46066 \\
c_{11}^{B} & =\frac{2}{5-\sqrt{5}}=\frac{5+\sqrt{5}}{10} \approx 0.72361 \\
c_{12}^{B} & =\frac{2}{\sqrt{5}} \approx 0.89443 .
\end{aligned}
$$

The Boston matching is therefore given by

$$
m^{B}(l, c, s)= \begin{cases}1 & \text { if } s=1, l \in\left[0 ; \beta_{1}\right] \text { and } c_{h} \in\left[0 ; c_{11}^{B}\right] \\ 1 & \text { if } s=2, l \in\left[\beta_{1} ; 5 / 6\right] \text { and } c_{h} \in\left[0 ; c_{1}^{B}\right] \\ 1 & \text { if } s=3, l \in\left[0 ; \beta_{1}\right] \text { and } c_{h} \in\left[c_{11}^{B} ; 1\right] \\ 1 & \text { if } s=3, l \in\left[\beta_{1} ; 5 / 6\right] \text { and } c_{h} \in\left[c_{12}^{B} ; 1\right] \\ 1 & \text { if } s=3, l \in[5 / 6 ; 1] \\ 0 & \text { otherwise }\end{cases}
$$

and the total transportation costs generated by this Boston matching are

$$
\begin{aligned}
T^{B}= & \frac{5+\sqrt{5}}{10} \int_{0}^{\frac{5-\sqrt{5}}{6}}|1 / 3-l| d l+\frac{5-\sqrt{5}}{10} \int_{0}^{\frac{5-\sqrt{5}}{6}}|1-l| d l+ \\
& \frac{2}{\sqrt{5}} \int_{\frac{5-\sqrt{5}}{6}}^{\frac{5}{6}}|2 / 3-l| d l+\frac{\sqrt{5}-2}{\sqrt{5}} \int_{\frac{5-\sqrt{5}}{6}}^{\frac{5}{6}}|1-l| d l+\int_{\frac{5}{6}}^{1}|1-l| d l \\
= & -\frac{5-\sqrt{5}}{18}+\frac{5+\sqrt{5}}{90}+\frac{17 \sqrt{5}}{180}+\frac{35-14 \sqrt{5}}{72}+\frac{1}{72} \\
= & \frac{5}{18}-\frac{\sqrt{5}}{30} \approx 0.20324
\end{aligned}
$$


As expected these transportation costs satisfy

$$
T^{*}=\frac{1}{6} \approx 0.16667<T^{B} \approx 0.20324<T^{S}=\frac{28}{135} \approx 0.20741,
$$

because the households living in $[0.46066 ; 1 / 2]$ apply at the more promising $s_{2}$ as they should in the social optimum.

Again, the possibility that the children may have to travel long distance to the school diminishes the value of the left locations. The equilibrium prices in the Boston equilibrium with the zero lump sum tax are therefore given by

$$
\begin{aligned}
& p^{B}(l)= 1+0.20324-E\left[T^{B}(l)\right] \\
& E\left[T^{B}(l)\right]= \begin{cases}0.51760-l & \text { if } l \in[0 ; 1 / 3] \\
0.035191+0.44721 \cdot l & \text { if } l \in[1 / 3 ; 0.46066] \\
0.70186-l & \text { if } l \in[0.46066 ; 2 / 3] \\
-0.49071+0.78885 \cdot l & \text { if } l \in[2 / 3 ; 5 / 6] \\
1-l & \text { if } l \in[5 / 6 ; 1]\end{cases}
\end{aligned}
$$

The comparison of the (reservation) price functions of the efficient, the stable and the Boston solution illustrates the efficiency gains and losses of the three solutions. In all three solutions the households at the city center do not pay transportation costs so that the prices at $l=1$ reflect the equilibrium utilities in the expected order. In the efficient matching the households are assigned to the optimal school with certainty so that the households at location $1 / 3$ and $2 / 3$ also have no transportation costs and also pay the same highest price $p_{s}=7 / 6$, while the other households are compensated for this with lower prices. Both the stable and the Boston matching are inefficient, because some households travel away from the city center, while others are rejected at their favorite school. Consequently the peaks of the reservation price functions are lower than efficient school specific housing price. At $s_{1}=1 / 3$ the peak of the Boston price function is the lowest, because the rejected households do not have the chance to apply at school 2 . This is not always the case, as the acceptance probability at school 1 is higher 
in the Boston solution. At the second peak the order is reversed, because the acceptance probability is higher under the Boston than under the stable mechanism, while the option for the rejected households is, of course, the same under both mechanisms.

While there is always unnecessary back traveling in the stable allocation the Boston solution may even be efficient. To demonstrate this we modify the example changing the school locations to $(1 / 2,3 / 4,1)$ and the capacities to $(1 / 4,3 / 8,3 / 8)$. As in section 3 an efficient matching is defined by

$$
m^{*}(l, c, s)= \begin{cases}1 & \text { if } l \in\left[\kappa_{s-1} ; \kappa_{s}\right] \\ 0 & \text { otherwise }\end{cases}
$$

but now every matching which avoids traveling away from the city center leads to the same aggregate expected transportation costs of $T^{*}=(1 / 2-1 / 8) / 4+$ $3(3 / 4-7 / 16) / 8+3(1-13 / 16) / 8=12 / 128+15 / 128+9 / 128=36 / 128$. The Boston matching in this case is characterized by

$$
m^{B}(l, c, s)= \begin{cases}1 & \text { if } s=1, l \in[0 ; 0.5] \text { and } c_{h} \in[0 ; 0.5] \\ 1 & \text { if } s=2, l \in[0.5 ; 0.875] \\ 1 & \text { if } s=3, l \in[0 ; 0.5] \text { and } c_{h} \in(0.5 ; 1] \\ 1 & \text { if } s=3, l \in[0.875 ; 1] \\ 0 & \text { otherwise. }\end{cases}
$$

so that also no child has to travel away from the city center. The expected transportation costs are, as expected, $T^{B}=9 / 32=0.28125$, and hence efficient. The stable matching, on the other hand, is

$$
m^{S}(l, c, s)= \begin{cases}1 & \text { if } s=1, l \in[0 ; 0.625] \text { and } c_{h} \in[0 ; 0.4] \\ 1 & \text { if } s=2, l \in[0 ; 0.625] \text { and } c_{h} \in(0.4 ; 0.714] \\ 1 & \text { if } s=2, l \in[0.625 ; 0.875] \text { and } c_{h} \in[0 ; 0.714] \\ 1 & \text { if } s=3, l \in[0 ; 0.875] \text { and } c_{h} \in(0.714 ; 1] \\ 1 & \text { if } s=3, l \in[0.875 ; 1] \\ 0 & \text { otherwise. }\end{cases}
$$

so that the inefficient transportation costs are $T^{S}=669 /(7 \cdot 5 \cdot 64)=0.29866$. This inefficiency of the stable solution arises because $40 \%$ of the households 
living in $[0.5 ; 0.625]$ and $71 \%$ of the households living in $[0.75 ; 0.875]$ travel away from the city center.

\section{Early Information}

The efficiency gain of BM is only realized as long as the households cannot foresee the consequences of their application decisions. If the households know all rankings before they submit their preferences the critical cost levels are sufficient information to avoid unsuccessful applications so that every instability of the final outcome generates the potential for a successful deviation in the second stage. Consequently the stable matching is also reached in the BM equilibrium.

Theorem 3 (Interim equivalence) Suppose all households know their characteristics $\left(l_{h}, c_{h}\right)$ before the application procedure starts, then a BM equilibrium outcome coincides with the stable solution constructed by DA. Moreover, all households who are not assigned to school $S$ under the stable matching, that is all $h \notin\left(\mu^{S}\right)^{-1}(S)$, list the school which they are assigned to in the stable matching first, $R_{h}^{B}(1)=\mu^{S}(h)$.

Proof: Suppose the households apply such that the Boston mechanism generates an unstable solution, that is, there are households $h^{\prime}=\left(l^{\prime}, c^{\prime}\right)$ matched to school $s^{\prime}$ and $h^{\prime \prime}=\left(l^{\prime \prime}, c^{\prime \prime}\right)$ matched to school $s^{\prime \prime}$ such that $c^{\prime}<c^{\prime \prime}$ and $\left|L_{s^{\prime}}-l^{\prime}\right|>\left|L_{s^{\prime \prime}}-l^{\prime}\right|$. Let household $h^{\prime}$ change the application strategy and turn to school $s^{\prime \prime}$ in the first round. Since $s^{\prime \prime}$ accepts $h^{\prime \prime}$ its capacity cannot be filled with better students in the first round (i.e. $c_{s i}^{B} \geq c^{\prime \prime}$ ), hence $h^{\prime}$ would be accepted in school $s^{\prime}$ and can thus increase his payoff with the deviation.

Suppose there is a household $h$ located at $l_{h}<1-K_{S}$ with $\mu^{S}(h)=s$ for some $s<S$ who does not list $s$ first. As this household is eventually assigned to $s$ there is empty capacity in school $s$ after round 1 , i.e. $c_{s 1}^{B}=1$. Moreover, 
there are households $h^{\prime}$ located at $l_{h^{\prime}} \in\left(b_{s-1} ; b_{s}\right)$ whose $c_{h^{\prime}}$ is so large that $\mu^{S}\left(h^{\prime}\right)=S$. In equilibrium such a household $h^{\prime}$ must be assigned to school $S$ so that it could deviate successfully from the equilibrium strategy listing school $s$ first.

This equivalence result is interesting, because it favors DA over BM. The two procedures yield equivalent outcomes, but this leaves us with the usual disadvantages of BM. While DA constructs the stable solution from the preferences revealed truthfully by the households (and schools) BM requires that rational households compute (or learn) the critical costs and then submit their best replies.

We can push the same question one step further and ask what happens if the households have some information about the priorities $c_{h}$ already when they chose their locations. This is particularly reasonable if these priorities are determined by the individual education costs, because the individual characteristics as well as the schools' preferences are to a large extent determined by the social attributes of the households so that it is not entirely convincing to assume that the preference of the schools come as a surprise. We will now show how the (equivalent) matching schemes DA and BM influence the final allocation if there is full information about the $c_{h}$ from the very beginning of the two-stage procedure.

Under full information households with a large $c_{h}$ know already in the first stage that they are not successful in an over-demanded school so that these households have an incentive to move towards the city center. This diminishes the competitive pressure at the outskirts and makes the housing prices there interesting for households with low $c_{h}$. We can therefore expect a sorting mechanism according to the $c_{h}$. The resulting segregation of types is interesting, because efficiency does not impose any restriction on the allocation of households to locations, because every distribution of household characteristics $\left(l_{h}, c_{h}\right)$ leads to an efficient equilibrium if the planner commits to implement the efficient matching $m^{*}$. Moreover, this segregation comes 
with unequal treatment. The households with higher priorities in the school matching mechanism gain using their advantage to live in neighborhoods close to schools which do not accept children of disadvantaged households. In this sense the school matching result reverts the Tiebout (1956) hypothesis where sorting by types is driven by efficiency in the favour of all households.

Theorem 4 (Ex ante social segregation) Suppose all households know their priorities $c_{h}$ before the housing market clears, then BM as well as DA generate an efficient equilibrium $\left(\mu^{F I}, p^{F I}\right)$ in which low priority households live closer to the center, $\mu^{F I}(h)>\mu^{F I}\left(h^{\prime}\right) \Rightarrow c_{h}>c_{h^{\prime}}$, while the equilibrium prices are continuous and satisfy

$$
\begin{aligned}
p^{F I}(l) & =\phi_{s}-t \cdot\left|l-L_{s}\right| \text { for } l \in\left[\kappa_{s-1} ; \kappa_{s}\right] \\
\phi_{s+1}-\phi_{s} & =t \cdot\left(\left(L_{s+1}-L_{s}\right)-2 \cdot \min \left\{\left(\kappa_{s}-L_{s}\right), 0\right\}\right)
\end{aligned}
$$

All households whose children are assigned to the same school s have the same equilibrium payment $u_{h}^{F I}=\phi_{s}+\tau$, while households whose children are assigned to a school closer to the center are worse off, $\phi_{1}<\phi_{2}<\ldots<\phi_{S}$.

The proof of this theorem is also deferred to the appendix.

Example In the example introduced above the stable allocation produced by both DA and BM if the priorities are known before the preferences are submitted was computed in section 4 . Thus it remains to compute the equilibrium prices and payoffs in the efficient allocation if the priorities are known already before the locations are chosen. In this case the locations of households satisfy $l_{h}=c_{h}$, the equilibrium matching is given by $\mu^{*}(h)=s$ if $l \in[(s-1) / 3 ; s / 3]$, and the equilibrium prices satisfy $p^{*}(l)=l$ with $\phi_{2}-\phi_{1}=\phi_{3}-\phi_{2}=1 / 3$, so that there is a clear advantage from segregation for high priority households. 


\section{Conclusion}

Reading the actual discussion on mechanism design and market engineering an economist who grew up when general equilibrium analysis was still an issue may get the impression that the literature focusses too much on the little problem at hand and too little on the context of these problems. In this paper we tried to demonstrate that this worry may not be entirely mislead. We tried to put the matching mechanism as one particular widely discussed rationing scheme into perspective by understanding it as one last step of the solution to a larger economic allocation problem. In a simple urban context we could show that efficiency is indeed context dependent and that convincing criteria for a well-functioning matching mechanism may not be as convincing if we look at them from the contextual perspective.

We tried to make this point as simple as possible so that the model which we introduced for this purpose is too simplistic for a serious application. Nevertheless, we think that not only the central message of the paper is more general than the assumptions we impose, but that our approach also allows generalizations which have the potential to contribute to an interesting discussion of actual policy issues.

On the demand side our model oversimplifies the relevant characteristics of the true school choice problem. In reality schools differ by their qualities and households have very strong preferences for these differences. Our model allows to include this aspect into the willingness to pay for a location in the neighborhood of a certain school. Of course such an extension would complicate our analysis severely, but this exercise may contribute interesting new aspects to the ongoing discussion about the desirable properties of a school matching mechanism, and contrary of the recent attempts to identify further refinements which distinguish between different efficient outcomes for instance on the basis of cardinal utility or bounded rationality ${ }^{16}$ our approach would allow a discussion solely on the grounds of established efficiency crite-

\footnotetext{
${ }^{16}$ As, for instance, Abdulkadiroglu et.al. (2011) or Apesteguia and Ballester (2012).
} 
ria.

On the supply side our assumptions are even further away from a serious attempt to describe reality, since our model assumes that school capacities are fixed, while both quality and quantity of schooling are clearly variable at least in the long run. In order to reach an efficient solution in our model it would be enough to extend the schooling capacities at the outskirts and reduce them in the center of our town, and in the resulting model of horizontal quality differentiation there would be no serious matching problem left. However, if location is not the only quality characteristic of a school the supply of schools becomes a complicated positive and normative issue. A serious attempt to analyze the consequences of the generation and distribution of economic rents by an imperfect matching mechanism would have to embed the matching mechanism in a much more general Tiebout-type regional model, but this task is left for future research.

\section{References}

Abdulkadiroglu, A., Y.-K. Che and Y. Yasuda (2011). "Resolving Conflicting Preferences in School Choice: The 'Boston Mechanism' Reconsidered." American Economic Review 101(1), 1-14.

Abdulkadiroglu, A. and T. Sönmez (2003). "School Choice: A Mechanism Design Approach." The American Economic Review 93(3), 729-747.

Abdulkadiroglu, A., P.A. Pathak and A.E. Roth (2005). "The New York City High School Match." The American Economic Review, Papers and Proceedings, 95(2), 364-367.

Abdulkadiroglu, A., P.A. Pathak, A.E. Roth, and T. Sonmez (2005). "The Boston Public School Match." The American Economic Review, Papers and Proceedings, 95(2), 368-371.

Alonso, W. (1964). "Location and land use.", Cambridge: Harvard University Press. 
Apesteguia, J. and M.A. Ballester (2012). "Welfare of Naive and Sophisticated Players in School Choice." Economics Letters, 115(2), 172-174.

Arnott, R.J. and J.E. Stiglitz (1979). "Aggregate Land Rents, Expenditure on Public Goods, and Optimal City Size." The Quarterly Journal of Economics 93(4), 471-500.

Avery, C., C. Jolls, R.A. Posner, and A.E. Roth (2001). "The Market for Federal Judicial Law Clerks." University of Chicago Law Review, 68(3), 793-902.

Bewley T.F. (1981). "A Critique of Tiebout's Theory of Local Public Expenditures." Econometrica, 49(3), pp.713-740.

Braun, S., N. Dwenger and D. Kübler (2010). "Telling the Truth May Not Pay Off: An Empirical Study of Centralized University Admissions in Germany." The B.E. Journal of Economic Analysis and Policy, 10(1), Article 22.

Bulow, J. and J. Levin (2006). "Matching and Price Competition." The American Economic Review 96(3), 652-668.

Kamecke, U. (1998). "Wage Formation in a Centralized Matching Market." International Economic Review 39(1), 33-53.

Oates, W.E. (1969). "The Effects of Property Taxes and Local Public Spending on Property Values: An Empirical Study of Tax Capitalization and the Tiebout Hypothesis." The Journal of Political Economy, 77(6), 957971.

Roth, A.E. (1984). "The Evolution of the Labor Market for Medical Interns and Residents: A Case Study in Game Theory." Journal of Political Economy, 92, 991-1016.

Roth, A.E. (1991). "A Natural Experiment in the Organization of EntryLevel Labor Markets: Regional Markets for New Physicians and Surgeons in the United Kingdom." The American Econmic Review, 81(3), 415-440.

Roth, A.E. and M. Sotomayor (1990). "Two-Sided Matching: A Study in 
Game-Theoretic Modeling and Analysis." Econometric Society Monograph Series, Cambridge University Press.

Roth, A.E., T. Sönmez and M.U. Ünver (2004). "Kidney Exchange." Quarterly Journal of Economics, 119(2), 457-488.

Roth, A.E., T. Sönmez and M.U. Ünver (2005). "Pairwise Kidney Exchange." Journal of Economic Theory, 125(2), 151-188.

Roth, A.E. and X. Xing (1994). "Jumping the Gun: Imperfections and Institutions Related to the Timing of Market Transactions." The American Economic Review, 84(4), 992-1044.

"The Prize in Economic Sciences 2010 - Advanced Information." Nobelprize.org. Nobel Media AB 2014. Web. 29 Aug 2014. <http://www. nobelprize.org/nobel_prizes/economic-sciences/laureates/2010/advanced.html> Tiebout, Charles (1956). "A Pure Theory of Local Expenditures." Journal of Political Economy, 64(5), 416-24.

\section{Appendix: Proof of Theorems 2 and 4}

\subsection{Equilibrium Properties (Theorem 2)}

Existence of the critical school $\bar{s}^{B}$ : If $c_{s 1}^{B}=1$ then all $h$ with $l_{h} \in\left[\bar{L}_{s-1} ; \bar{L}_{s}\right]$ select $R_{h}^{B}(1)=s$ so that $c_{s 1}^{B}<1$ whenever $K_{s}>\bar{L}_{s}-$ $\bar{L}_{s-1}$, in particular for all $s \leq \bar{s}$ by assumption $(1)$. Since $\lambda(\mathcal{H})=1$ there must be $s$ with $c_{s 1}^{B}=1$ so let $\bar{s}^{B}$ be the smallest such $s$. Let $\widetilde{\mathcal{S}}=$ $\left\{s \in\left\{s^{B}+1, \ldots, S\right\} \mid c_{1 s}^{B}<1\right\}, \widetilde{\mathcal{S}}^{c}=\left\{s \in\left\{s^{B}+1, \ldots, S\right\} \mid c_{1 s}^{B}=1\right\}$, and suppose that $\widetilde{\mathcal{S}} \neq \emptyset$ so that $\bar{s}^{B}>\bar{s}$ implies that $\lambda\left(\left\{h \mid R_{h}^{B}(1) \in \widetilde{\mathcal{S}}\right\}\right)>$ $\sum_{s \in \widetilde{\mathcal{S}}} K_{s}$ even though $\sum_{s \in \widetilde{\mathcal{S}}} K_{s}>\sum_{s \in \widetilde{\mathcal{S}}} \bar{L}_{s}-\bar{L}_{s-1}$ by (1). This first round excess demand for $s \in \widetilde{\mathcal{S}}$ does not come from $l_{h}<L_{\bar{s}^{B}}$, because a first round application $R_{h}^{B}(1)=\bar{s}^{B}$ is always successful while a later $(i>1)$ applica- 
tion $R_{h}^{B}(i)<\bar{s}^{B}$ never succeeds, so that a household with $R_{h}^{B}(1) \in \widetilde{\mathcal{S}}$ and $l_{h}<\bar{L}_{s^{B}}$ could reduce his expected transportation cost with a deviation to a strategy $R_{h}^{\prime}$ with $R_{h}^{\prime}(1)=\bar{s}^{B}$. The excess demand does also not come from neighborhoods of $s \in \widetilde{\mathcal{S}}^{c}$, since a household $h$ with $l_{h} \in\left[\bar{L}_{s-1} ; \bar{L}_{s}\right]$ for some $s \in \widetilde{\mathcal{S}}^{c}$ will always play $R_{h}^{B}(1)=s_{h}$. Consequently, $\left\{h \mid R_{h}^{B}(1) \in \widetilde{\mathcal{S}}\right\} \subset$ $\cup_{s \in \mathcal{S}^{\prime}}\left[\bar{L}_{s-1} ; \bar{L}_{s}\right]$ and hence $\lambda\left(\left\{h \mid R_{h}^{B}(1) \in \widetilde{\mathcal{S}}\right\}\right) \leq \sum_{s \in \widetilde{\mathcal{S}}} \bar{L}_{s}-\bar{L}_{s-1}$. This completes a contradiction.

Ring structure: We show the following: if two households $h_{1}$ and $h_{2}$ located at $l_{h_{1}}=l_{1}<l_{2}=l_{h_{2}}$ who list schools $R_{h_{1}}^{B}(1)=s_{2}$ and $R_{h_{2}}^{B}(1)=s_{1}$ located at $L_{1}<L_{2}$ on their first ranks, then the two don't lose if they play each others' strategies and they gain unless they are both located to the left of $L_{1}$, that is, in equilibrium this only happens if $l_{1}<l_{2} \leq L_{1}<L_{2}$ and if the expected transportation costs satisfy $T_{h_{1}}\left(R^{B}\right)=T_{h_{1}}\left(R^{B} \mid R_{h_{1}}=R_{h_{2}}^{B}\right)$ and $T_{h_{2}}\left(R_{h_{2}}^{B}\right)=T_{h_{2}}\left(R \mid R_{h_{2}}=R_{h_{1}}^{B}\right)$.

Denote the strategies of household $h_{i}$ by $R_{i}$, its conditionally expected transportation cost for $c \geq \bar{c}$ by $T_{i}^{\bar{c}}=T_{i}^{c \geq \bar{c}}$, the critical cost levels by $c_{s_{i} 1}^{B}=$ $c_{i 1}^{B}$, and let $\bar{L}=\left(L_{1}+L_{2}\right) / 2$. If $l_{2}>\bar{L}_{\bar{s}^{B}-1}$ the argument above implies that $h_{2}$ is assigned to his first ranked school at $L_{1} \geq L_{\bar{s}^{B}}$. Consequently $h_{1}$ would never rank $L_{2}>L_{1}$ first and the claim follows.

If $l_{2} \leq \bar{L}_{\bar{s}^{B}-1}$ both households have to travel towards the center if they are not successful with $R_{h}^{B}(1)$ so that $T_{1}^{\bar{c}}=T_{2}^{\bar{c}}+t\left|l_{2}-l_{1}\right|$. If in addition $\bar{L} \leq l_{1}$ then $c_{s_{1} 1}^{B}>c_{s_{2} 1}^{B}$ because the household at $l_{2}>l_{1}$ could reduce the expected transportation costs by exchanging the ranks of the schools $s_{1}$ and $s_{2}$ in $R_{2}^{B}$ otherwise. Using this together with $\left|L_{1}-l_{2}\right|=\left|l_{2}-l_{1}\right|+\left|L_{1}-l_{1}\right|$, the equilibrium condition $T_{1}\left(R^{B}\right) \leq T_{1}\left(R^{B} \mid R_{2}^{B}\right)$, and the triangle inequality 
$\left|L_{2}-l_{2}\right| \leq\left|L_{2}-l_{1}\right|+\left|l_{2}-l_{1}\right|$ we derive the following contradiction:

$$
\begin{aligned}
T_{2}\left(R^{B}\right)= & c_{11}^{B} t\left|L_{1}-l_{2}\right|+\left(1-c_{11}^{B}\right) T_{2}^{c_{11}^{B}}\left(R^{B}\right) \\
= & c_{11}^{B} t\left(\left|L_{1}-l_{1}\right|+\left|l_{2}-l_{1}\right|\right)+\left(1-c_{11}^{B}\right)\left(T_{1}^{c_{11}^{B}}\left(R^{B}\right)-t\left|l_{2}-l_{1}\right|\right) \\
= & \left(2 c_{11}^{B}-1\right) t\left|l_{2}-l_{1}\right|+T_{1}\left(R^{B} \mid R_{2}^{B}\right) \\
\geq & \left(2 c_{11}^{B}-1\right) t\left|l_{2}-l_{1}\right|+T_{1}\left(R^{B}\right) \\
= & \left(2 c_{11}^{B}-1\right) t\left|l_{2}-l_{1}\right|+t c_{21}^{B}\left|L_{2}-l_{1}\right|+\left(1-c_{21}^{B}\right) T_{1}^{c_{21}^{B}}\left(R^{B}\right) \\
\geq & \left(2 c_{11}^{B}-1\right) t\left|l_{2}-l_{1}\right|+t c_{21}^{B}\left(\left|L_{2}-l_{2}\right|-\left|l_{2}-l_{1}\right|\right) \\
& +\left(1-c_{21}^{B}\right)\left(T_{2}^{c_{21}^{B}}\left(R^{B}\right)+t\left|l_{2}-l_{1}\right|\right) \\
= & \left(2 c_{21}^{B}-2 c_{21}^{B}\right) t\left|l_{2}-l_{1}\right|+T_{2}\left(R^{B} \mid R_{1}^{B}\right) \\
> & T_{2}\left(R^{B} \mid R_{1}^{B}\right)
\end{aligned}
$$

If, on the other hand, $l_{1} \leq \bar{L}$ we use $\left|L_{2}-l_{1}\right|=\left|l_{2}-l_{1}\right|+\left|L_{2}-l_{2}\right|$, the equilibrium condition $T_{2}\left(R^{B} \mid R_{1}^{B}\right) \geq T_{2}\left(R^{B}\right)$ and the triangle inequality $\left|L_{1}-l_{1}\right| \leq\left|L_{1}-l_{2}\right|+\left|l_{2}-l_{1}\right|$ to get

$$
\begin{aligned}
T_{1}\left(R^{B}\right) & =c_{21}^{B} t\left|L_{2}-l_{1}\right|+\left(1-c_{21}^{B}\right) T_{1}^{c_{21}^{B}}\left(R^{B}\right) \\
& =c_{21}^{B} t\left(\left|l_{1}-l_{2}\right|+\left|L_{2}-l_{2}\right|\right)+\left(1-c_{21}^{B}\right)\left(T_{2}^{c_{21}^{B}}\left(R^{B} \mid R_{1}^{B}\right)+t\left|l_{1}-l_{2}\right|\right) \\
& =T_{2}\left(R^{B} \mid R_{1}^{B}\right)+c_{21}^{B} t\left|l_{1}-l_{2}\right|+\left(1-c_{21}^{B}\right) t\left|l_{1}-l_{2}\right| \\
& \geq T_{2}\left(R^{B}\right)+t\left|l_{1}-l_{2}\right| \\
& =c_{11}^{B} t\left(\left|L_{1}-l_{2}\right|+\left|l_{1}-l_{2}\right|\right)+\left(1-c_{11}^{B}\right)\left(T_{2}^{c_{11}^{B}}\left(R^{B}\right)+t\left|l_{1}-l_{2}\right|\right) \\
& \geq c_{11}^{B} t\left|L_{1}-l_{1}\right|+\left(1-c_{11}^{B}\right) T_{1}^{c_{11}^{B}}\left(R^{B} \mid R_{2}^{B}\right) \\
& =T_{1}\left(R^{B} \mid R_{2}^{B}\right)
\end{aligned}
$$

Since the equilibrium strategy minimizes transportation costs the inequalities 
must holds with "=". For $l_{2}>L_{1}$ the triangle inequality and hence the second inequality is strict so that we must have $l_{2} \leq L_{1}$ and the claim follows.

Monotonicity of $c_{s 1}^{B}$ : Suppose that $c_{11}^{B} \geq c_{21}^{B}$. Take a household $h$ with $l_{h} \leq \bar{L}_{1}$ and denote the strategy which exchanges the ranking of schools 1 and 2 by $R_{h}^{1 \leftrightarrows 2}$. If $R_{h}^{B}(1)=2$ then $R_{h}^{1 \leftrightarrows 2}$ satisfies $T_{h}^{B}\left(R_{h}^{1 \leftrightarrows 2}\right) \leq T_{h}^{B}(R)$ (with strict inequality if $l_{h}<\bar{L}_{1}$ or $\left.c_{11}^{B}>c_{21}^{B}\right)$, because $T_{h}^{c \geq c_{11}}\left(R_{h}^{1 \leftrightarrows 2}\right)=$ $T_{h}^{c \geq c_{11}}(R) \geq\left|l_{h}-L_{3}\right|, T_{h}^{c \leq c_{21}}\left(R_{h}^{1 \leftrightarrows 2}\right)=\left|L_{1}-l_{h}\right| \leq\left|L_{2}-l_{h}\right|=T_{h}^{c \leq c_{21}}(R)$ and $T_{h}^{c_{11} \geq c \geq c_{21}}\left(R_{h}^{1 \leftrightarrows 2}\right)=\left|L_{1}-l_{h}\right| \leq\left|l_{h}-L_{3}\right| \leq T_{h}^{c_{11} \geq c \geq c_{21}}(R)$. Therefore the ring structure property above implies that $R_{h}^{B}(1)=1$ for all households $h$ with $l_{h}<\bar{L}_{1}$. Since $c_{11}^{B} \geq c_{21}^{B}$ is only possible if there are at least as many first round applicants per place in school 1 as in school 2, assumption (1) implies that $\lambda\left(\left\{h \mid R_{h}^{B}(1)=2\right\}\right) \geq \bar{L}_{2}-\bar{L}_{1}$. Thus there must be households $h$ located at $l_{h}>\bar{L}_{2}$ with $R_{h}^{B}(1)=2$. This, in turn, is only possible if $c_{21}^{B}>c_{31}^{B}$, because otherwise a deviation to $R_{h}^{2 \leftrightarrows 3}$ would be profitable for these households. Iterating this argument we get $c_{s 1}>c_{s+11}$ for all $s$ and hence $\sum_{s=1}^{S} \lambda\left(A_{s 1}^{B}\right)>\sum_{s=1}^{S} K_{s}=1$, a contradiction to the assumption that the set of all households has measure 1.

Finally, suppose that $c_{11}<c_{21}$ and $c_{21} \geq c_{31}$. A repetition of the argument above for the second ring gives a contradiction unless $c_{21}=1$, and the iteration of this argument completes the proof of our equlibrium properties.

Efficiency: Monotonicity implies that the ring borders $b_{s}^{B}$ satisfy $b_{s}^{B} \leq$ $\bar{L}_{s}$ with a strict inequality if $c_{s+12}^{B}<c_{s+11}^{B}$ (i.e., for instance, if $s<\bar{s}^{B}-1$ ), because the household types $c \in\left(c_{s 1}^{B} ; c_{s+11}^{B}\right)$ have to travel further than to $s+1$ if they are not successful with $R_{h}^{B}(1)=s$, while the remaining typies $c \in\left[c_{s+11}^{B} ; 1\right]$ are also not better off after the first round rejection. If $b_{s}^{B}<\bar{L}_{s}$ 
there is some $\varepsilon>0$ such that households $h \in\left(\bar{L}_{s}-\varepsilon ; \bar{L}_{s}\right)$ would have applied for school $s$ under DA so that $c_{s 1}^{S}$ of them would travel outward to school $s$, while they travel to school $s+1$ towards the center under BS. Since all capacities are filled there are $\varepsilon \cdot c_{s 1}^{S}$ households $h$ with $l_{h} \leq b_{s}^{B}$ who are assigned to school $s$ under BS, while they were assigned to school $s^{\prime}>s$ under DA. This exchange of households saves at least the total transportation cost of $\Delta T=\varepsilon \cdot t \cdot c_{s 1}^{S} \cdot\left(\bar{L}_{s}-b_{s}^{B}-\varepsilon\right)$.

\subsection{Existence (Theorem 2)}

Fixed Point Argument Throughout the following we restrict our search for an equilibrium strategy by reducing the complexity of potential strategies drastically. For $s<S$ we assume that all households in the ring $h \in\left[b_{s-1} ; b_{s}\right]$ list school $s$ first, that they list on the following ranks only the $S-s-2$ schools $s^{\prime}$ which are located closer to but not at the center (i.e. $s<s^{\prime}<S$ ), and that they list school $S$ last. Moreover, we enumerate these $J_{s}=(S-s-1)$ ! rankings of the form $\widetilde{R}_{s j}=$ $\left(s, \widetilde{R}_{s j}(2), \ldots, \widetilde{R}_{s j}(S-s-1), S\right)$, and interpret $\psi_{s j}$ as the share of households of every measurable subset of locations of $\left[b_{s-1} ; b_{s}\right]$ who, independently of the cost characteristic $c_{h}$, submit the $j$-th of these modified (partial) rankings $\widetilde{R}_{s j} \cdot{ }^{17}$

To apply a fixed point argument we construct a u.h.c. correspondence $(\beta, \psi) \rightarrow(B, \Psi)$ of right ring borders and of reduced best replies as follows. The vectors $(\beta, \psi)$ and the subsets $(B, \Psi)$ of potential right ring borders and modified partially mixed strategies are taken from the closed and convex set

$$
\mathfrak{B} \times \mathfrak{P}=\left[0 ; \bar{L}_{s}\right]^{S-2} \times\left\{p \in[0 ; 1]^{(S-2) \times J_{s}} \mid \sum_{j=1}^{J_{s}} \psi_{s j}=1\right\} .
$$

\footnotetext{
${ }^{17}$ This way we avoid the introduction of the equivalent mixed strategies $\psi_{s j}$ played by all households in the ring so that we don't have to modify the Boston algorithm.
} 
For each $(\beta, \psi) \in \mathfrak{B} \times \mathfrak{P}$ we define the image $(B, \Psi)(\beta, \psi) \subset \mathfrak{B} \times \mathfrak{P}$ of our correspondence in three steps as follows.

First, we construct ring borders recursively by $b_{1}(\beta)=\beta_{1}$ and

$$
b_{s}(\beta)=\max \left\{\beta_{s} ; b_{s-1}(\beta)\right\} \text { for } s=1 \ldots S-2,
$$

and define the expected transportation cost function for each ring $s<S$, for each vector of critical costs $c^{B}=\left(c_{s i}^{B}\right)_{s<S}^{i<S}$, each mixed ranking $p_{s} \in \mathfrak{P}_{s}$ of the remaining schools $s+1 \ldots J_{s}$, and each location $l \in[0 ; 1]$ as

$$
T_{s}\left(c^{B}, p_{s}, l\right)=\sum_{j=1}^{J_{s}} p_{s j} \cdot t \cdot\left(\sum_{i=1}^{S-s}\left(c_{\widetilde{R}_{s j}(i), i}^{B}-c_{\widetilde{R}_{s j}(i-1), i-1}^{B}\right) \cdot\left|l-L_{\widetilde{R}_{s j}(i)}\right|\right)
$$

with the understanding that $\widetilde{R}_{s j}(0)=0$ and $c_{00}^{B}=0$. It is obvious that $T_{s}$ is continuous.

Second, we denote the critical costs constructed by the Boston algorithm according to (5) for the modified matching problem by $\widetilde{c}_{s i}^{B}(\beta, \psi)$ and use this to define the strategy image of our fixed point correspondence for each $s$ as the set of best replies at the right border

$$
\Psi_{s}(\beta, \psi)=\arg \min _{p_{s} \in \mathfrak{P}_{s}} T_{s}\left(\widetilde{c}^{B}(\beta, \psi), p_{s}, \beta_{s}\right) .
$$

Third, we construct the set of locations at which the households are indifferent between listing $s$ first and listing some other alternative $s^{\prime}$ first. For this purpose we define upper and lower contours of candidate locations for each $s$ and each $(\beta, \psi)$ by

$\mathfrak{L}_{s}^{\leq}(\cdot)=\left\{l \in\left[0 ; \bar{L}_{s}\right] \mid T_{s}\left(\widetilde{c}^{B}(\cdot), \psi_{s}, l\right) \leq T_{s^{\prime}}\left(\widetilde{c}^{B}(\cdot), \psi_{s^{\prime}}, l\right)\right.$ for all $\left.s^{\prime}>s\right\}$

$\mathfrak{L}_{s}^{\geq}(\cdot)=\left\{l \in\left[0 ; \bar{L}_{s}\right] \mid T_{s}\left(\widetilde{c}^{B}(\cdot), \psi_{s}, l\right) \geq T_{s^{\prime}}\left(\widetilde{c}^{B}(\cdot), \psi_{s^{\prime}}, l\right)\right.$ for some $\left.s^{\prime}>s\right\}$

and derive the corresponding set of potential borders for each ring $s$ by

$$
B_{s}(\beta, \psi)= \begin{cases}\mathfrak{L}_{s}^{\leq}(\beta, \psi) \cap \mathfrak{L}_{s}^{\geq}(\beta, \psi) & \text { if } \mathfrak{L}_{s}^{\leq}(\cdot) \cap \mathfrak{L}_{s}^{\geq}(\cdot) \neq \emptyset \\ \{0\} & \text { if } \mathfrak{L}_{s}^{\leq}(\beta, \psi)=\emptyset \\ \left\{\bar{L}_{s}\right\} & \text { if } \mathfrak{L}_{s}^{\geq}(\beta, \psi)=\emptyset .\end{cases}
$$


Notice that the critical costs $\widetilde{c}_{s i}^{B}(\beta, \psi)$ vary continuously with $(\beta, \psi)$ since the number of applicants and hence of rejections in each iteration varies continuously with $(\beta, \psi)$. Consequently, the strategy image $\Psi(\beta, \psi)$ is u.h.c. and convex valued. Notice further that the mean value theorem for the continuous $T_{s}$ implies that $\mathfrak{L}_{s}^{\leq} \cap \mathfrak{L}_{s}^{\geq} \neq \emptyset$ if $\mathfrak{L}_{s}^{\leq} \neq \emptyset$ and $\mathfrak{L}_{s}^{\geq} \neq \emptyset$ so that $B_{s}(\beta, \psi)$ is well defined. By construction we have $L_{\widetilde{R}_{s j}(i)}>\bar{L}_{s}$ for all $i=2, \ldots$ so that

$$
\begin{aligned}
l<l^{\prime} \leq \bar{L}_{s} & \Rightarrow\left|l-L_{\widetilde{R}_{s j}(i)}\right|=\left|l-l^{\prime}\right|+\left|l^{\prime}-L_{\widetilde{R}_{s j}(i)}\right| \Rightarrow \\
T_{s}\left(c^{B}, p_{s}, l\right)-T_{s}\left(c^{B}, p_{s}, l^{\prime}\right) & =c_{s 1}^{B} t\left(\left|l-\bar{L}_{s}\right|-\left|l^{\prime}-\bar{L}_{s}\right|\right)+\left(1-c_{s 1}^{B}\right) t\left(l^{\prime}-l\right) \\
& \leq\left(l^{\prime}-l\right) \cdot t .
\end{aligned}
$$

For $s^{\prime}>s$ with $L_{s^{\prime}}>\bar{L}_{s}$ the same argument gives

$$
l<l^{\prime} \leq \bar{L}_{s} \Rightarrow T_{s^{\prime}}\left(c^{B}, p_{s^{\prime}}, l\right)-T_{s^{\prime}}\left(c^{B}, p_{s^{\prime}}, l^{\prime}\right)=t \cdot\left(l^{\prime}-l\right) .
$$

Combining the two we get the monotone differences

$$
T_{s}\left(c^{B}, p_{s}, l\right)-T_{s^{\prime}}\left(c^{B}, p_{s^{\prime}}, l\right) \leq T_{s}\left(c^{B}, p_{s}, l^{\prime}\right)-T_{s^{\prime}}\left(c^{B}, p_{s^{\prime}}, l^{\prime}\right)
$$

so that there are $b_{s}^{\leq}$and $b_{s}^{\geq}$with $\mathfrak{L}_{s}^{\leq}(\beta, \psi)=\left[0 ; b_{s}^{\leq}\right]$and $\mathfrak{L}_{s}^{\geq}(\beta, \psi)=\left[b_{s}^{\geq} ; \bar{L}_{s}\right]$ if the sets are non-empty. Consequently $B_{s}(\beta, \psi) \in\left\{\left[b_{s}^{\geq} ; b_{s}^{\leq}\right],\{0\},\left\{\bar{L}_{s}\right\}\right\}$ is convex valued. Moreover, $B(\beta, \psi)$ is u.h.c., because (8) garantees that 0 is the last and $\bar{L}_{s}$ the first transportation cost minimizing candidate border, in the sense that $\left(\beta^{n}, \psi^{n}\right) \rightarrow(\beta, \psi)$ with $\mathfrak{L}_{s}^{\leq}\left(\beta^{n}, \psi^{n}\right)=\emptyset$ and $\mathfrak{L}_{s}^{\leq}(\beta, \psi) \neq \emptyset$ implies that $\mathfrak{L}_{s}^{\leq}(\beta, \psi)=\{0\} \subset \mathfrak{L}_{s}^{\geq}(\beta, \psi)$, while $\left(\beta^{n}, \psi^{n}\right) \rightarrow(\beta, \psi)$ with $\mathfrak{L}_{s}^{\geq}\left(\beta^{n}, \psi^{n}\right)=\emptyset$ and $\mathfrak{L}_{s}^{\geq}(\beta, \psi) \neq \emptyset$ implies that $\mathfrak{L}_{s}^{\geq}(\beta, \psi)=\left\{\bar{L}_{s}\right\} \subset \mathfrak{L}_{s}^{\leq}(\beta, \psi)$.

Since the mapping $(\beta, \psi) \rightarrow(B, \Psi)$ is convex valued and u.h.c there must be a fixed point $\left(\beta^{*}, \psi^{*}\right) \in(B, \Psi)\left(\beta^{*}, \psi^{*}\right)$. and it remains to show that this fixed point is a Nash equilibrium of the matching game. 
Equilibrium To facilitate the notation let $c_{i s}^{*}=c_{i s}^{B}\left(\beta^{*}, \psi^{*}\right)$ and $T_{s}^{*}(l)=$ $T_{s}\left(c^{*}, \psi_{s}^{*}, l\right)$. The ring borders have two important properties. First, they satisfy $b_{s}\left(\beta^{*}\right)=\beta_{s}^{*}$ : if $b_{s}\left(\beta^{*}\right)=b_{s-1}\left(\beta^{*}\right)>\beta_{s}^{*}$ in $(6)$ then $c_{s 1}^{*}=1$ (as there are no applicants for $s)$; hence $\left(\bar{L}_{s-1} ; \bar{L}_{s}\right) \cap \mathfrak{L}_{s}^{\geq}\left(\beta^{*}, \psi^{*}\right)=\emptyset \Rightarrow \beta_{s}^{*}=\bar{L}_{s}>\beta_{s^{\prime}}^{*}$ for all $s^{\prime}<s$. Secondly, the monotonicity argument used in the last paragraph of 8.1 is valid for $\beta_{s}^{*}$ as well so that there must be a school $\bar{s}^{*}$ such that $c_{11}^{*}<\ldots<c_{\dot{s}^{*} 1}^{*}=c_{\bar{s}^{*}+11}^{*}=c_{S-11}^{*}=1$ : if $c_{11}^{*} \geq c_{21}^{*}$ then $\beta_{1}^{*}=\bar{L}_{1}$; assumption (1) implies that $\beta_{2}^{*}-\beta_{1}^{*}>\bar{L}_{2}-\bar{L}_{1}$ so that $\beta_{2}^{*}=\bar{L}_{2}$ and hence $c_{21}^{*} \geq c_{31}^{*}$; iterating this argument gives a contradiction to $c_{11}^{*}<1$ so that $c_{11}^{*}<c_{21}^{*}$; if $c_{11}^{*}<c_{21}^{*}$ and $c_{21}^{*} \geq c_{31}^{*}$ we have $\beta_{2}^{*}=\bar{L}_{2}$ and hence $c_{21}^{*} \geq c_{31}^{*}$; iterating this argument gives a contradiction unless $c_{21}^{*}=1$, and so on.

We have to show that the deviation from the equlibrium strategy to some pure strategy $R_{h}^{\prime}$ of all schools does not pay for any household $h$ located at $l_{h} \in[0 ; 1]$. To see this note first that $\psi_{s}^{*}$ solves the optimization problem

$$
\psi_{s}^{*} \in \arg \min _{p_{s} \in \mathfrak{P}_{s}} T_{s}\left(c^{*}, p_{s}, l_{h}\right)
$$

for all households located at $l_{h}<\bar{L}_{\bar{s}^{*}}$ : since all schools $s<\bar{s}^{*}$ are filled in the first round $\left(c_{s i}^{*}=0\right.$ for all $i>1$ ) households who are not successful with their first rank must travel to some $s \geq \bar{s}^{*}$; thus

$$
\begin{aligned}
& T_{s}\left(c^{*}, \psi_{s}, l_{h}\right) \\
= & t \cdot c_{s 1}^{*} \cdot\left|l_{h}-L_{s}\right|+t \cdot \sum_{j=1}^{J_{s}} \psi_{s j}\left(\sum_{i=2}^{S-s}\left(c_{\widetilde{R}_{s j}(i), i}^{*}-c_{\widetilde{R}_{s j}(i-1), i-1}^{*}\right) \cdot\left|L_{\widetilde{R}_{s j}(i)}-l_{h}\right|\right) \\
= & t \cdot c_{s 1}^{*} \cdot\left|l_{h}-L_{s}\right|+\left(1-c_{s 1}^{*}\right)\left(\beta_{s}^{*}-l_{h}\right) \\
& +t \cdot \sum_{j=1}^{J_{s}} \psi_{s j}\left(\sum_{i=2}^{S-s}\left(c_{\widetilde{R}_{s j}(i), i}^{*}-c_{\widetilde{R}_{s j}(i-1), i-1}^{*}\right) \cdot\left|L_{\widetilde{R}_{s j}(i)}-\beta_{s}^{*}\right|\right) \\
= & T_{s}\left(c^{*}, \psi_{s}, \beta_{s}^{*}\right)+t \cdot c_{s 1}^{*} \cdot\left(\left|l_{h}-L_{s}\right|-\left|\beta_{s}^{*}-L_{s}\right|\right)+t \cdot\left(1-c_{s 1}^{*}\right)\left(\beta_{s}^{*}-l_{h}\right)
\end{aligned}
$$


implies that the difference $\left(T_{s}\left(c^{*}, \psi_{s}, l\right)-T_{s}\left(c^{*}, \psi_{s}, \beta_{s}^{*}\right)\right)$ is independent of $\psi_{s}$ so that $\psi_{s}^{*}$ minimizes $T_{s}\left(c^{*}, \psi_{s}, l_{h}\right)$, too.

The fixed point strategies assign households $l_{h} \geq \bar{L}_{\bar{s}^{*}-1}$ to there closest schools so that these households cannot deviate successfully from their equilibrium strategies. The argument above implies that households who select $R_{h}^{\prime}(1)=s^{\prime}<\bar{s}^{*}$ as their first rank cannot do better than to continue with $\psi_{s}^{*}$ (or one of the pure strategies which receive a positive probability in $\psi_{s}^{*}$ ). The optimality of $\psi_{s}^{*}$ and the construction of $\beta_{s}^{*}$ with the first case of (7) imply further that a household $l_{h} \in\left[\beta_{s-1}^{*} ; \beta_{s}^{*}\right]$ can never gain from a deviation to $s^{\prime}>s$ so that suppose that households $h$ with $l_{h} \in\left[\beta_{s-1}^{*} ; \beta_{s}^{*}\right]$ and $s<\bar{s}^{*}$ can deviate successfully to a strategy $R$ with $R_{h}^{\prime}(1)=s^{\prime}<s$. In this case $l_{h} \in \mathfrak{L}_{s^{\prime}}^{\geq}\left(\beta^{*}, \psi^{*}\right)$ so that there is some $s^{\prime \prime}>s^{\prime}$ with $T_{s^{\prime \prime}}\left(c^{*}, \psi^{*}, l_{h}\right) \leq T_{s^{\prime}}\left(c^{*}, \psi^{*}, l_{h}\right)$ so that there is another successful deviation to some $R^{\prime \prime}$ with $R^{\prime \prime}(1)=s^{\prime \prime}$. Thus $s^{\prime \prime}>s$ is not possible, while $s^{\prime \prime}<s$ implies that there is another successful deviation to some $R^{\prime \prime \prime}$ with $R^{\prime \prime \prime}(1)=s^{\prime \prime \prime}>s^{\prime \prime}$. Iterating this argument gives a successful deviation to a strategy $\widehat{R}$ with $\widehat{R}(1)=s$ and hence a contradiction to the optimality of $\psi^{*}$

\subsection{Social Segregation (Theorem 4)}

Notice first that

$$
\begin{aligned}
p^{F I}(l) & =\phi_{s}-t \cdot\left|l-L_{s}\right| \text { for } l \in\left[\kappa_{s-1} ; \kappa_{s}\right] \\
\phi_{s+1}-\phi_{s} & =t \cdot\left(\left(L_{s+1}-L_{s}\right)-2 \cdot \min \left\{\left(\kappa_{s}-L_{s}\right), 0\right\}\right)
\end{aligned}
$$

define a continous price function: assumption (1) implies $\bar{L}_{s}>\kappa_{s}$. For $L_{s} \geq \kappa_{s}(10)$ reduces to $\phi_{s+1}-\phi_{s}=t \cdot\left(L_{s+1}-L_{s}\right)>0$ so that

$$
\begin{aligned}
\lim _{l \uparrow \kappa_{s}} p^{F I}(l) & =\phi_{s}-t \cdot\left(L_{s}-\kappa_{s}\right) \\
& =\phi_{s+1}-t \cdot\left(L_{s+1}-\kappa_{s}\right)=\lim _{l \downarrow \kappa_{s}} p^{F I}(l)
\end{aligned}
$$


while for $L_{s}<\kappa_{s}(10)$ becomes $\phi_{s+1}-\phi_{s}=t \cdot\left(\left(L_{s+1}-L_{s}\right)-2 \cdot\left(\kappa_{s}-L_{s}\right)\right)=$ $2 \cdot t \cdot\left(\bar{L}_{s}-\kappa_{s}\right)>0$ and so

$$
\begin{aligned}
\lim _{l \uparrow \kappa_{s}} p^{F I}(l) & =\phi_{s}-t \cdot\left(\kappa_{s}-L_{s}\right) \\
& =\phi_{s+1}-t \cdot\left(L_{s+1}-\kappa_{s}\right)=\lim _{l \downarrow \kappa_{s}} p^{F I}(l) .
\end{aligned}
$$

Next we argue that the location choices $l_{h}=c_{h}$ generate the equilibrium allocation specified above. As better households live further away from the center both DA and BM construct $\mu^{F I}(h)$. Moreover, no household has an incentive to deviate: the households' payments $u_{h}^{F I}=\phi_{s}+\tau$ coincide inside each school ring $\left[\kappa_{s-1} ; \kappa_{s}\right]$, so that it is not possible to gain by choosing another location there; moving to a location in another ring and applying to the same school does not pay, because $p^{F I}(\cdot)$ is continuous with $\left|\left(p^{F I}\right)^{\prime}(\cdot)\right| \leq t$; and moving to a location in another ring and applying to another school does also not pay, because applying to a school $s<\mu^{F I}(h)-1$ is not successful, while applying to a school $s>\mu^{F I}(h)$ does not pay as $\phi_{s}>\phi_{\mu^{F I}(h)}$.

To see that every equilibrium has these properties notice first that free mobility implies that the equilibrium price function $p^{F I}$ must be continuous, because otherwise a household could gain from changing to a cheaper location close to his equilibrium location and still being assigned to the same school. For the same reason the equilibrium payoffs of all households $h \in\left(\mu^{F I}\right)^{-1}(s)$ assigned to the same school must coincide, $u_{h}^{F I}=\phi_{s}+\tau$. Finally, this argument implies that $p^{F I}(l)-p^{F I}\left(L_{s}\right)=t\left|L_{s}-l\right|$ (and hence (10)) for every location $l \in\left[l_{h} ; L_{s}\right]$ between $L_{s}$ and a location $l_{h}$ chosen as residence by some household $h \in\left(\mu^{F I}\right)^{-1}(s)$, because otherwise either the households living close to $l$ (if ' $>$ ' holds) or the household living close to $l_{h}$ (if ' $<$ ' holds) could deviate successfully.

Take $s=S$, then by assumption (1) $p^{F I}(l)=\phi_{S}-t \cdot\left|l-L_{S}\right|$ holds 
for the locations $l \geq \kappa_{S-1}$. Households living at $l \in\left[\kappa_{S-1} ; \bar{L}_{S-1}\right]$ would prefer $S-1$, so that all households assigned to $S-1$ are preferred by the school. Moreover, free mobility implies that this holds also for all other $h \in\left(\mu^{F I}\right)^{-1}(S)$. Iterating this argument for $s=S-1, \ldots, 1$ gives the social segregation by schools $h \in\left(\mu^{F I}\right)^{-1}(s)$ and $h^{\prime} \in\left(\mu^{F I}\right)^{-1}(s-1) \Rightarrow c_{h}>c_{h^{\prime}}$.

To derive the social segregation of neighborhoods note that all households located at $l \geq \bar{L}_{S-1}$ are assigned to school $S$ by DA so that these locations are occupied by $h \in\left(\mu^{F I}\right)^{-1}(S)$. Suppose that all households $h$ located at $l_{h} \geq \bar{L}_{\widehat{s}}$ are assigned to schools $s>\widehat{s}$ so that these schools have a total capacity of $\bar{L}_{\widehat{s}}-\kappa_{\widehat{s}}>0$ left.

If $L_{\widehat{s}}<\kappa_{\widehat{s}}$ there must be $l, l^{\prime} \in\left[L_{\widehat{s}} ; \bar{L}_{\widehat{s}}\right]$ such that $h_{l} \in\left(\mu^{F I}\right)^{-1}(\widehat{s})$ and $h_{l^{\prime}} \in\left(\mu^{F I}\right)^{-1}\left(s^{\prime}\right)$ for some $s^{\prime}>\widehat{s}$. As $p^{F I}(\cdot)$ is decreasing up to $l$ and increasing from $l^{\prime}$ onward there must be a boundary $b_{\widehat{s}}$ separating the two groups such that $l_{h}>b_{\widehat{s}} \Leftrightarrow \mu^{F I}(h)>\widehat{s}$. As households located at $l<b_{\widehat{s}}$ are not assigned to a school $s>\widehat{s}$ we have $b_{\widehat{s}}=\kappa_{\widehat{s}}<\bar{L}_{\widehat{s}}$ so that all households $h$ located at $l_{h} \in\left[\bar{L}_{\widehat{s}-1} ; \bar{L}_{\widehat{s}}\right]$ are assigned to school $\widehat{s}$ and $\phi_{s+1}-\phi_{s}=t$. $\left(\left(L_{s+1}-L_{s}\right)-2 \cdot\left(\kappa_{s}-L_{s}\right)\right)$ (i.e. $\left.(11)\right)$.

If, on the other hand, $L_{\widehat{s}} \geq \kappa_{\widehat{s}}$ the interval $\left[L_{\widehat{s}} ; \bar{L}_{\widehat{s}}\right]$ has not enough locations to host all households which have to be assigned to schools $s>\widehat{s}$. Thus there must be households $h \in\left(\mu^{F I}\right)^{-1}(s)$ with $l_{h} \leq L_{\widehat{s}}$ for some $s>\widehat{s}$. Consequently $p^{F I}(\cdot)$ is increasing on $\left[\bar{L}_{\widehat{s}-1} ; \bar{L}_{\widehat{s}}\right]$ with $h \in\left(\mu^{F I}\right)^{-1}(\widehat{s}) \Rightarrow l_{h} \leq$ $L_{\widehat{s}}$ so that $\phi_{s+1}-\phi_{s}=t \cdot\left(L_{s+1}-L_{s}\right)$ (i.e.(11)) and $l_{h} \geq \bar{L}_{\widehat{s}-1} \Rightarrow \mu^{F I}(h) \geq \widehat{s}$ 